\title{
1 Multidrug transporters and organic anion transporting \\ 2 polypeptides protect insects against the toxic effects of 3 cardenolides
}

4

5 Simon C. Groen ${ }^{\mathrm{a}{ }^{*}}$, Erika R. LaPlante ${ }^{\mathrm{a}, \mathrm{b}}$, Nicolas M. Alexandre ${ }^{\mathrm{a}, \mathrm{b}}$, Anurag A. Agrawal ${ }^{\mathrm{c}, \mathrm{d}}$,

6 Susanne Dobler ${ }^{\mathrm{e}}$, Noah K. Whiteman ${ }^{\mathrm{a}, \mathrm{b}^{*}}$

7

$8 \quad{ }^{a}$ Department of Ecology and Evolutionary Biology, University of Arizona, Tucson, AZ

9 85721, USA

$10{ }^{b}$ Department of Integrative Biology, University of California, Berkeley, 3040 Valley Life

11 Sciences Building, Berkeley, CA 94720, USA

$12{ }^{\mathrm{c}}$ Department of Ecology and Evolutionary Biology, Cornell University, Ithaca, New

13 York 14853, USA

$14{ }^{\mathrm{d}}$ Department of Entomology, Cornell University, Ithaca, New York 14853, USA

15 e Molecular Evolutionary Biology, Zoological Institute, Biocenter Grindel, Universität

16 Hamburg, Martin-Luther-King Pl. 3, 20146 Hamburg, Germany

17 * Corresponding authors: Department of Biology, Center for Genomics and Systems

18 Biology, New York University, New York, New York 10003, USA. Email:

19 sg189@nyu.edu (S.C. Groen); Department of Integrative Biology, University of

20 California, Berkeley, 3040 Valley Life Sciences Building, Berkeley, CA 94720, USA.

21 Email: whiteman@ berkeley.edu (N.K. Whiteman).

$22{ }^{1}$ Present address: Department of Biology, Center for Genomics and Systems Biology,

23 New York University, New York, New York 10003, USA

24

25 Keywords: Drosophila melanogaster; cardenolide; Na/K-ATPase; multidrug transporter /

26 P-glycoprotein; organic anion transporting polypeptide; herbivore. 


\section{Abstract (248 words)}

In the struggle against dietary toxins, insects are known to employ target site

31 insensitivity, metabolic detoxification, and transporters that shunt away toxins.

32 Specialized insects across six taxonomic orders feeding on cardenolide-containing plants

33 have convergently evolved target site insensitivity via specific amino acid substitutions in

34 the $\mathrm{Na} / \mathrm{K}$-ATPase. Nonetheless, in vitro pharmacological experiments have suggested a

35 role for multidrug transporters (Mdrs) and organic anion transporting polypeptides

36 (Oatps), which may provide a basal level of protection in both specialized and non-

37 adapted insects. Because the genes coding for these proteins are evolutionarily conserved

38 and in vivo genetic evidence in support of this hypothesis is lacking, here we used

39 wildtype and mutant Drosophila melanogaster (Drosophila) in capillary feeder (CAFE)

40 assays to quantify toxicity of three chemically diverse, medically relevant cardenolides.

41 We examined multiple components of fitness, including mortality, longevity, and

42 LD50, and found that, while the three cardenolides each stimulated feeding (i.e., no

43 deterrence to the toxin), all decreased lifespan, with the most apolar cardenolide having

44 the lowest LD50 value. Flies showed a clear non-monotonic dose response and

45 experienced high levels of toxicity at the cardenolide concentration found in plants. At

46 this concentration, both Mdr and Oatp knockout mutant flies died more rapidly than

47 wildtype flies, and the mutants also experienced more adverse neurological effects on

48 high-cardenolide-level diets. Our study further establishes Drosophila as a model for the

49 study of cardenolide pharmacology and solidifies support for the hypothesis that

50 multidrug and organic anion transporters are key players in insect protection against

51 dietary cardenolides.

52

53 


\section{Introduction}

The insect herbivores adapted to withstand the toxic effects of cardiac glycosides (cardenolides and bufodienolides) produced by milkweeds (Asclepias spp.), foxglove (Digitalis spp.), oleander (Nerium oleander), lily of the valley (Convallaria majalis) and many other plant species comprise a model system to study convergent evolution at the molecular, physiological, morphological and behavioral levels (Agrawal et al., 2012; Dobler et al., 2015; Stern, 2013; Storz, 2016). Much molecular and genetic work has focused on putatively adaptive amino acid substitutions in the $\mathrm{Na} / \mathrm{K}$-ATPase that block cardenolides from binding to this essential pump (Dalla et al., 2013; Dalla and Dobler, 2016; Dobler et al., 2012, 2015; Zhen et al., 2012). However, recent findings indicate that the amino acid substitutions in the $\mathrm{Na} / \mathrm{K}$-ATPase may not be the only adaptations to reduce sensitivity to dietary cardenolides. Larvae of the lepidopteran species Empyreuma pugione, Daphnis nerii, and Euploea core are specialized on cardenolide-bearing plants and do not have known substitutions in the $\mathrm{Na} / \mathrm{K}$-ATPase. Indeed, in vitro analysis of their enzyme indicates a high level of sensitivity to cardenolides (Petschenka et al., 2012, 2013, 2015; Petschenka and Dobler, 2009). Additionally, some generalists are able to cope very well feeding on cardenolide-containing plants (Agrawal, unpublished; Züst and Agrawal, 2016). These results indicate that the substitutions in the Na/K-ATPase are not necessarily required for life on cardenolide-producing plants and that alternative mechanisms including metabolic detoxification (Marty and Krieger, 1984), and efflux carriers may be important (Petschenka et al., 2013).

Of these alternative mechanisms that confer resistance to cardenolides (reviewed by Agrawal et al., 2012; Dobler et al., 2015), one set of adaptations involves the peritrophic membrane in the midgut (Barbehenn 1999, 2001), and epithelial diffusion barriers such as septate junctions in both midgut and perineurium (blood (or hemolymph)-brain-barrier, BBB). These structures can form efficient protective 
81 mechanisms against hydrophilic or polar cardenolides such as ouabain that do not cross membranes passively (Figure 1) (Petschenka et al., 2013; Petschenka and Agrawal, 2016; Züst and Agrawal, 2016). In this way, polar cardenolides are to some extent prevented from reaching the nervous tissue where they could otherwise have adverse neurological effects (Armstrong et al., 2011; Ashmore et al., 2009; Schubiger et al., 1994; Xia et al., 1997, 1998). However, moderate-to-highly lipophilic or apolar cardenolides, such as digoxin and digitoxin, do cross membranes passively and must be removed through active transport when they penetrate the midgut and threaten to pass the BBB (Figure 1). Two main gene families involved in active cardenolide transport mechanisms have been characterized through in vitro physiological experiments, which we describe next.

Multidrug transporters (Mdrs), alternatively described as P-glycoproteins (P-gps), are B-subfamily ABC transporters (Dermauw and Van Leeuwen, 2014). Mdrs are present in the BBB of all animals (Hindle and Bainton, 2014), and are active diffusion barriers for the apolar cardenolide digoxin in insects and vertebrates alike (Gozalpour et al., 2013; Petschenka et al., 2013). Expression of several ABC transporters including Mdrs is also enriched in the Malpighian tubules (Chahine and O'Donnell, 2009; Dow and Davies, 2006). Transport capacity increases dramatically upon exposure to organic toxins (Chahine and O'Donnell, 2009), and is coordinated with the activity of xenobiotic detoxification mechanisms (Chahine and O'Donnell, 2011). Furthermore, staining with Mdr-specific antibodies and tissue-specific measurements of gene expression have shown that Mdrs are present in the midgut of both cardenolide-encountering and non-adapted insects alike (Dobler et al., 2015; Petschenka et al., 2013). This suggests that they could be targets for adaptive evolution during the specialization process on cardenolide-bearing plants.

Organic anion transporting polypeptides (Oatps) are also expressed in the BBB and midgut (Hagenbuch and Stieger, 2013; Hindle and Bainton, 2014). In addition, some Oatps show high expression levels in the Malpighian tubules (Torrie et al., 2004), where 
they are expressed alongside $\mathrm{ABC}$ transporters such as the Mdrs, and detoxification enzymes (e.g. cytochrome P450s and glutathione-S-transferases), and play a major role in the metabolism and excretion of xenobiotics and endogenous solutes (Dow and Davies, 2006). Although the importance of Oatps has not been tested functionally in cardenolideadapted insects, in vitro reverse genetic experiments on Malpighian tubules of Drosophila found that a subset of Oatps protect the $\mathrm{Na} / \mathrm{K}$-ATPase from interference by the polar cardenolide ouabain (Torrie et al., 2004). Thus, these Oatps are also potential targets for adaptive evolution during the transition to a cardenolide-containing diet.

Further work established that the monarch butterfly (Danaus plexippus) and large milkweed bug (Oncopeltus fasciatus) might possess unidentified carriers that regulate the balance between cardenolide efflux and sequestration in these specialist herbivores (Frick and Wink, 1995; Meredith et al., 1984; Scudder and Meredith, 1982; Seiber et al., 1980). Given their conserved biological function, members of the Mdr and Oatp families are prime candidates for adaptive evolution (Dobler et al., 2015; Petschenka and Agrawal, 2016). Together with work showing the protective effects of Mdrs and Oatps against plant-derived toxins and pesticides (Dermauw and Van Leeuwen, 2014; Seabrooke and O'Donnell, 2013; Torrie et al., 2004), these findings have led to the hypothesis that the broad-substrate Mdrs and Oatps might have a general role in excluding plant toxins at the gut membrane and BBB, and in excreting toxins at the Malpighian tubules (Dobler et al., 2015). The high level of amino acid sequence conservation across animals suggests that these transporters could provide some resistance against cardenolides in most insects. The level of resistance might then be enhanced through adaptation during specialization on cardenolide-producing host plants (Dobler et al., 2015).

Here we tested the hypothesis that Mdrs and Oatps provide a basal level of resistance against a set of chemically diverse cardenolides in a non-adapted insect. We chose Drosophila as a model system for studying the function of these transporters in response to cardenolides because of the genetic tools and behavioral assays available 
135 (Deshpande et al., 2014; Groen and Whiteman, 2016). Furthermore, populations of a 136 close relative of Drosophila, D. subobscura have been reared from decaying cardenolide137 producing plants (Kearney, 1983) and evolved a derived Na/K-ATPase gene copy with 138 amino acid substitutions that confer resistance to cardenolides (Pegueroles et al., 2016). 139 The three cardenolides we selected (Figure 1) have been used in the clinic for decades in 140 treatments of heart conditions (Ambrosy et al., 2014). Furthermore, both Mdrs and Oatps 141 have a large influence on the pharmacokinetics of drugs in cancer therapy and treatments 142 of other ailments (Borst and Schinkel, 2013; Dean et al., 2001; Hagenbuch and Stieger, 143 2013; Obaidat et al., 2012). In light of the importance of cardenolides, and Mdr and Oatp 144 transporters in human medicine there is a surprising dearth of information on cardenolide 145 pharmacology/toxicology and $\mathrm{Mdr}$ and Oatp function in one of the prime model 146 organisms for biomedical research, Drosophila (Wangler et al., 2015).

147 We first established that cardenolide concentrations as found in plants are 148 relatively toxic to a non-adapted insect, and that apolar cardenolides have more 149 consistently toxic effects than polar cardenolides. We then found that Drosophila 150 transporter knockout mutants have shorter lifespans on diets containing biologically 151 relevant levels of cardenolides than wildtype flies, and that these mutant flies succumb 152 after intake of lower amounts of cardenolides. Finally, transporter knockout mutants 153 suffer more adverse acute neurological effects from high dietary cardenolide levels than 154 wildtype flies. Although previous studies suggest that some degree of caution is 155 warranted in linking transporter expression to transport of a specific substrate (Chahine et 156 al., 2012a and -b), our findings point to an important role for Mdrs and Oatps in 157 protecting insects against cardenolides, and provide further evidence for these 158 transporters as candidates for adaptation to a cardenolide-containing diet. 


\section{Materials and methods}

\subsection{Chemicals}

165

The cardenolides ouabain ( $\geq 95 \%$ purity), digoxin ( $\geq 95 \%$ purity), and digitoxin ( $\geq 92 \%$ purity) - listed in decreasing order of polarity (Frick and Wink, 1995) - were obtained from Sigma-Aldrich (St Louis, MO, USA). The cardenolides were dissolved in

168 the solvent dimethyl sulfoxide (DMSO; $\geq 99 \%$ purity; Santa Cruz Biotechnology, Dallas, 169 TX, USA), which was subsequently diluted to a final concentration of $0.1 \%$ DMSO with 170 a 5\% sucrose (weight / volume) fly food solution. The concentration of $0.1 \%$ DMSO was 171 chosen to be below the experimentally determined "no observed adverse effect level" of $1720.3 \%$ for Drosophila (Nazir et al., 2003). The cardenolide concentrations of the solutions 173 were confirmed with high-performance liquid chromatography (HPLC) as described by 174 Züst and Agrawal (2016). Information on the LD50 of the three cardenolides was found 175 in the NIH TOXNET ChemIDplus database (visited: 09/09/2016; oubain: 176 http://chem.sis.nlm.nih.gov/chemidplus/rn/630-60-4; digoxin:

177 http://chem.sis.nlm.nih.gov/chemidplus/rn/20830-75-5; digitoxin:

178 http://chem.sis.nlm.nih.gov/chemidplus/rn/71-63-6).

Lines of Drosophila melanogaster (Meigen) with P-elements resident in the 182 coding region of the following Mdr and Oatp genes were obtained from public sources: Mdr49 (PMdr49: $w^{1118}$; Mi\{ET1\}Mdr49 ${ }^{\mathrm{MB} 04959}$ ) (Bloomington Drosophila Stock Center 184 [BDSC], Indiana University, Bloomington, IN, USA; line 24312; Bellen et al., 2004); $185 M d r 50$ (PMdr50: $w^{1118} ; \mathrm{pBac}[\mathrm{pB}]\left\{w^{+\mathrm{mC}}\right\} M d r 50^{\mathrm{c} 00522}$ ) (Exelixis at Harvard Medical 186 School, Boston, MA, USA; line c00522; Bellen et al., 2011; Mayer et al., 2009; Parks et 187 al., 2004; Thibault et al., 2004); Mdr65 (PMdr65: $y^{1} w^{67 c 23}$; P\{SUPor-P $\} M d r 65^{\mathrm{KG} 08723}$ 
$188 \mathrm{ry}^{506}$ ) (BDSC; line 14757; Bellen et al., 2004; Mayer et al., 2009); Oatp30B (POatp30B:

$189 w^{1118} ;$ PBac $\{$ RB $\}$ Oatp30B ${ }^{\mathrm{e} 00405}$ ) (Exelixis at BDSC; line 17854; Bellen et al., 2004; Parks

190 et al., 2004; Thibault et al., 2004); Oatp33Eb (POatp33Eb: $y^{1} w^{*}$; $191 \mathrm{Mi}\{\mathrm{MIC}\}$ Oatp33Eb $b^{\mathrm{M} 103575}$ ) (BDSC; line 37058; Bellen et al., 2004); Oatp58Db 192 (POatp58Db: $y^{1} w^{*} ; \mathrm{Mi}\{$ MIC $\}$ Oatp58Db $^{\mathrm{M} 102785}$ ) (BDSC; line 36047; Bellen et al., 2004, 193 2011). See FlyBase (http://flybase.bio.indiana.edu/) for more information (dos Santos et 194 al., 2015). The line Oregon-R-C (OreR or "OR" in this study; BDSC; line 5) was used as 195 wildtype.

196

197 198

199

200

201

202

203

204

205

206

207

208

209

210

211

212

213

Fly stocks were maintained on cornmeal-agar-yeast medium (obtained from the University of Arizona Bio5 Media Facility) at $20^{\circ} \mathrm{C}$ under $16 \mathrm{~h}$ light: $8 \mathrm{~h}$ dark conditions at a relative humidity of $30 \%$.

\subsection{CAFE assay}

Fly longevity and food intake was measured using the CApillary FEeder (CAFE) assay (Deshpande et al., 2014; Ja et al., 2007). Three adult flies of the same sex from stock cultures at two days post eclosion were placed in each of four vials per treatment group. A sample size of $\mathrm{N}=4$ is sufficient to detect effect sizes of $20 \%$ in CAFE assays (Deshpande et al., 2014). The two-day period ensured all flies were mated. The flies were supplied daily with two capillaries containing $c a .7 \mu \mathrm{L} 5 \%$ sucrose solution with or without cardenolides as described in section 2.1. This ensured ad libitum food availability, and consumption was measured as described by Ja and co-workers (2007). No yeast was added to the sucrose solutions, as this would introduce an additional factor that could influence food intake (Lee et al., 2008; Vigne and Frelin, 2007, 2010). CAFE assays were performed in the same growth room conditions as reported for fly stock maintenance. 


\subsection{Stress sensitivity assay}

Fly stress sensitivity was measured using the bang-sensitive paralysis assay (Ganetzky and Wu, 1982). One adult fly from stock cultures at two days post eclosion was placed in each of four vials per treatment group. Over the next two days the flies

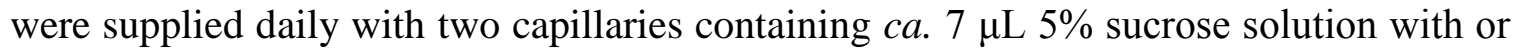
without cardenolides as described in section 2.1, and consumption was monitored. On each day the vials were vortexed once at maximum speed for $10 \mathrm{~s}$ on a standard laboratory vortexer and the time of recovery from paralysis and mortality recorded (Schubiger et al., 1994). Stress assays were performed in the same environmental conditions as reported for fly stock maintenance.

\subsection{Statistical analysis}

Statistical analysis was carried out using R (R Core Development Team, 2012). CAFE and stress sensitivity assays were analyzed using ANOVA with fly genotype, sex and diet as factors (CAFE assay), or with genotype and diet as factors (stress sensitivity). Subsequently, post hoc Tukey HSD tests were performed. Mortality data from stress sensitivity assays were analyzed using t-tests for binomial proportions.

\section{Results}

\subsection{Ouabain is relatively toxic at a cardenolide concentration found in plants}

We set up a 10x-step dilution CAFE assay with wildtype flies to test how Drosophila, an insect that does not normally encounter cardenolides, responds to a range of doses of these plant toxins. We restricted our experiment to the polar cardenolide ouabain because millimolar concentrations of more apolar cardenolides cannot be 
239 dissolved without exceeding DMSO's level of "no observed adverse effects" (Nazir et 240 al., 2003). As an anchor point for the dilution series, we calculated the cardenolide 241 concentration that is found in leaves of the common milkweed Asclepias syriaca. The 242 leaf concentration has been measured as $0.338 \pm 0.042 \mathrm{mg} / \mathrm{g}$ wet mass and therefore the 243 biologically relevant concentration an insect would encounter is ca. $0.5 \mu \mathrm{M}$ (Agrawal et 244 al., 2014). We set up our ouabain dilution series to include this concentration and cover a 245 million-fold range from $5 \mathrm{nM}$, which is 1,000x lower than the lowest previously studied, 246 orally administered concentration, to $5 \mathrm{mM}$, which is the highest previously studied 247 concentration (Ashmore et al., 2009; Beikirch, 1977).

Despite differences between male and female Drosophila in their response to 249 ouabain, both sexes showed a clear non-monotonic dose response (Figure 2A). 250 Surprisingly, the biologically relevant cardenolide concentration of $0.5 \mu \mathrm{M}$ (indicated 251 with an asterisk in Figure 2) was relatively more toxic than higher or lower doses. At 252 higher concentrations $(50 \mu \mathrm{M}$ and $0.5 \mathrm{mM})$ female flies were more susceptible to the 253 toxic effects of ouabain than males (Figure 2A). These observations were also reflected in 254 the LD50 expressed as $\mu \mathrm{L}$ of solution necessary to kill $50 \%$ of flies (Figure 2B).

\subsection{Cardenolide polarity influences toxicity}

257

Since the biologically relevant cardenolide concentration of $0.5 \mu \mathrm{M}$ showed a 258 considerable level of toxicity we continued performing CAFE assays using this 259 concentration. Cardenolides show a range of polarities, and apolar cardenolides generally 260 are more toxic than more polar cardenolides, since apolar cardenolides can passively 261 cross membranes (Agrawal et al., 2012). To test for this effect in Drosophila, we set up 262 an assay with three widely used, clinically important cardenolides (Gozalpour et al., 263 2013). Besides ouabain (polar), these were digoxin (slightly apolar) and digitoxin (highly 264 apolar) (Figure 1; Agrawal et al., 2012). 
The addition of any of the three cardenolides to the sucrose solution enhanced 266 feeding rates in females, but not in males (Figure 3A). These observations indicate that 267 none of the cardenolides had feeding deterrent effects in Drosophila, which is unlike what 268 has been observed with some other insect species that do not normally encounter 269 cardenolides in their diet (Akhtar and Isman, 2004; Sachdev-Gupta et al., 1993; Zhou et 270 al., 2010).

271 We observed that all three cardenolides reduced fly longevity, and that this 272 reduction was stronger in females than in males (Figure 3B). Further analysis revealed 273 that this was mainly driven by a stronger negative effect of ouabain on lifespan in 274 females than in males. Contrary to expectations, lifespan was reduced equally by all three 275 cardenolides in males, whereas the highly apolar digitoxin had a stronger negative effect 276 on female lifespan than digoxin (Figure 3B). However, the LD50 of both ouabain and 277 digoxin was higher than that of digitoxin either when calculated as ng of cardenolide 278 ingested per fly (Figure 3C), or as $\mu \mathrm{L}$ of cardenolide-laced sucrose solution ingested per 279 fly (Figure 3D).

The LD50 values for orally ingested cardenolides observed in our experiments on 281 Drosophila were comparable to those reported for vertebrate animals when expressed 282 relative to body weight for all three cardenolides (Table 1).

\subsection{Mdrs protect insects against cardenolides}

Mdr49, -50 and -65 have previously been identified as the Drosophila orthologues 286 of human P-glycoprotein (P-gp) (Gerrard et al., 1993; Wu et al., 1991). Apolar 287 cardenolides such as digoxin are P-gp substrates in vertebrates and insects (Gozalpour et 288 al., 2013; Petschenka et al., 2013). Mdr65 is mainly expressed in the BBB, Mdr50 in the 289 midgut, and Mdr49 in the Malpighian tubules. Mdr49 is also expressed at appreciable 
290 levels in the reproductive system, the BBB and the midgut (Supplemental Figure 1; 291 Brown et al., 2014; DeSalvo et al., 2014; Graveley et al., 2011; Mayer et al, 2009).

Even on a control diet not containing cardenolides, all three Mdr knockout mutant 293 lines suffered from reduced longevity in our experimental conditions (Supplemental 294 Figure 2). Altered lifespan has previously been observed for mutants in other $\mathrm{ABC}$ 295 transporters (Huang et al., 2014; Oxenkrug, 2010). Therefore, the effects of cardenolides 296 on fly lifespan were analyzed as the relative decrease in longevity caused by cardenolide 297 intake in knockout mutant flies compared to the decrease of lifespan in wildtype flies. Fly 298 sex had no effect and therefore data for male and female flies were analyzed together 299 (Supplemental Figure 2).

Digitoxin had a uniformly strong negative effect on lifespan across all genotypes 301 and both sexes, except for PMdr50 in which it had a weaker negative effect (Figure 4A). 302 However, the LD50 expressed as ng of digitoxin necessary to kill 50\% of flies was lower 303 for both the PMdr49 and PMdr65 mutants than for wildtype flies (Figure 4B). Again, the $304 P M d r 50$ mutant was the exception and digitoxin's LD50 for this mutant was more similar 305 to that for wildtype flies.

Digoxin had a more variable effect on fly lifespan than digitoxin. Whereas it 307 reduced lifespan relatively more strongly in PMdr65 mutants than in wildtype flies, 308 digoxin had the opposite effect in the PMdr49 and -50 mutants in which it reduced fly 309 lifespan less than in wildtype flies (Figure 4A). Despite these opposing effects of digoxin 310 on the relative lifespan of mutants, the LD50 expressed as ng of digoxin necessary to kill $31150 \%$ of flies was lower for all knockout mutants than for wildtype flies (Figure 4B).

To confirm the protective role of Mdrs against cardenolide toxicity, we performed 313 stress sensitivity (bang-sensitive paralysis) assays in which we vortexed vials with flies 314 for 10 s and measured mortality and the time to recovery from paralysis after flies were 315 fed a high dose of digoxin $(0.5 \mathrm{mM})$ for two days. The effects of digitoxin were not 316 tested in this assay since $0.5 \mathrm{mM}$ digitoxin could not be dissolved without exceeding 
317 DMSO's level of "no observed adverse effects" (Nazir et al., 2003). Both the PMdr50

318 and -65 mutants took longer to recover from vortexing than wildtype flies when they 319 were on the digoxin-containing diet (Figure 5A). In addition, digoxin-induced mortality 320 was enhanced for the PMdr65 knockout flies compared to the other genotypes (Figure $3215 \mathrm{~B})$.

\subsection{Oatps protect insects against cardenolides}

Oatps $30 \mathrm{~B}, 33 \mathrm{~Eb}$ and $58 \mathrm{Db}$ have been previously shown to bind to and transport 325 ouabain (Torrie et al., 2004). Oatp58Db is exclusively expressed in the Malpighian 326 tubules, where $O a t p 30 B$ and $-33 E b$ are also expressed (Torrie et al., 2004). Whereas 327 Oatp33Eb is additionally expressed in the midgut, Oatp30B is expressed at moderate 328 levels in most tissues (Supplemental Figure 3; Brown et al., 2014; Graveley et al., 2011).

The POatp33Eb knockout mutant line suffered from reduced longevity in our experimental conditions (Supplemental figure 4), thus, the effect of ouabain on fly lifespan was analyzed as the relative decrease in longevity caused by ouabain intake in 332 knockout mutant flies compared to the decrease of lifespan in wildtype flies. Fly sex had 333 no significant effect and data for male and female flies were analyzed together.

Ouabain reduced lifespan relatively more strongly in POatp30B mutant flies than 335 in wildtype flies, whereas it had no significant effect on relative lifespan in the 336 POatp33Eb and $-58 D b$ mutants (Figure 6A). Despite no effect of ouabain on the relative 337 lifespan of the latter two mutants, the LD50 expressed as ng of ouabain necessary to kill $33850 \%$ of flies was lower for all knockout mutants than for wildtype flies (Figure 6B).

To confirm the protective role of Oatps against ouabain toxicity, we performed 340 the stress sensitivity (bang-sensitive paralysis) assays in which flies were fed high doses 341 of ouabain $(0.5$ and $5 \mathrm{mM})$ for two days. Although no mutants took longer to recover 342 from vortexing than wildtype flies when they were on an ouabain-containing diet (Figure 
$3437 \mathrm{~A}$ and 7C), the ouabain-induced mortality was enhanced for the POatp58Db and $-33 E b$ 344 mutant flies compared to the wildtype flies (Figure 7B and 7D). Recovery time could not 345 be measured for the POatp33Eb mutant flies that consumed ouabain-containing food 346 (Figure 7C), since all mutant flies in the ouabain treatment groups died from intoxication 347 (Figure 7D).

\section{Discussion}

In this work we quantified the oral toxicity of three medically relevant cardenolides (ouabain, digoxin, digitoxin) in Drosophila, which typically does not encounter these toxins in its diet, and found that cardenolide concentrations as found in

354 plants are relatively poisonous to insects, with stronger negative effects for apolar than 355 for polar compounds. We also determined that the Mdr and Oatp transporters contribute 356 to protecting this insect to some extent from the harmful effects of cardenolides. These 357 are the first experiments in the complex, living organism that demonstrate the protective role of both of the previously presumptive groups of cardenolide transporters. Our experiments further establish Drosophila as a model for pharmacological and functional genetic studies of insect resistance to cardenolides (Groen and Whiteman, 2016).

In the high-precision CAFE assays, flies showed a clear non-monotonic dose response to ouabain and experienced relatively high levels of toxicity at the cardenolide 363 concentrations found in plants. Although a previous study observed a non-monotonic 364 dose response to ouabain as well (Ashmore et al., 2009), that study also found a positive 365 effect of low ouabain concentrations on fly longevity (hormesis), which we did not find 366 in our experiments. A likely explanation for this discrepancy is the difference in feeding 367 assays employed. Ashmore and colleagues administered ouabain solutions in filter paper on top of standard cornmeal-molasses agar medium. While it is difficult to quantify fly 
ouabain intake in that assay, flies that experience an increase in lifespan may have consumed sub-nanomolar concentrations of ouabain, below the lowest ouabain concentration that we used in our experiments using the more controlled CAFE assay.

The difference in feeding assays might also explain why Ashmore and colleagues did not observe that an intermediate concentration of ouabain can be more toxic to flies than higher concentrations, which we observed for the biologically relevant concentration of $0.5 \mu \mathrm{M}$ compared to the higher concentrations of 5 and $50 \mu \mathrm{M}$ (Ashmore et al., 2009). Despite the fact that Ashmore and co-workers added a concentration as high as $5 \mathrm{mM}$ ouabain to the filter papers that were placed on the agar medium, the flies may not have consumed an effective concentration higher than $0.5 \mu \mathrm{M}$. At this point we can only speculate as to why a concentration of $0.5 \mu \mathrm{M}$ ouabain would be more toxic to flies than $5 \mu \mathrm{M}$. Perhaps the ingestion of $0.5 \mu \mathrm{M}$ ouabain does not trigger profound immediate physiological responses in the insect, and the chronic ingestion of ouabain at this concentration would lead to a reduced lifespan. Higher concentrations might trigger a protective mechanism that is relatively effective at 5 and $50 \mu \mathrm{M}$, but might get overwhelmed at concentrations higher than $0.5 \mathrm{mM}$. More work would be necessary to elucidate if such a protective mechanism is indeed triggered, and if so, what contributions are made by Mdrs, Oatps and other actors.

An alternative explanation for why we did not observe a hormetic effect is that a CAFE assay offering food with carbon (sucrose), but no nitrogen source (such as yeast extract), might negate any lifespan-enhancing effect that low doses of ouabain might provide. Despite the fact that the lifespan of flies in our experiments was comparable to the lifespan observed in other studies that employed CAFE assays without a nitrogen source (Lee et al., 2008; Vigne and Frelin, 2007, 2010), such interactive effects of nutrient content and cardenolide toxicity on fly longevity remain a possible explanation.

At high doses of ouabain our results mirror previous results (Beikirch 1977, Ashmore et al. 2009). Beikirch (1977) administered ouabain by mixing it into cornmeal- 
agar-syrup medium, and observed that females were more susceptible to the toxic effects of ouabain than males, which we found as well. Moreover, as in both other studies, ouabain had a strong toxic effect at a concentration of $5 \mathrm{mM}$.

Our results are also in line with previous findings on the effects of polarity on cardenolide toxicity. As reviewed in detail by Agrawal and colleagues (2012), the polar ouabain in general tends to have a less severe impact on insects than the apolar digitoxin. Support for this has for example been found for larvae of the monarch butterfly, which are more negatively affected by digitoxin than by ouabain (Rasmann et al., 2009). This specialist herbivore also sequesters cardenolides for defense against attackers, and during sequestration it converts apolar cardenolides into more polar forms, while also preferentially sequestering polar cardenolides (Frick and Wink, 1995; Seiber et al., 1980). Although the polar cardenolides would likely not be as a good a defense against predators compared to apolar cardenolides, they can also be stored more conveniently since they do not passively cross membranes.

In the stress sensitivity and CAFE assays we observed acute and chronic toxic effects of cardenolides on flies, respectively. Reports of acute cardenolide toxicity in herbivorous insects are rare, but one study on the generalist herbivore Trichoplusia ni showed immediate adverse effects on this species (Dussourd and Hoyle, 2000). Studies on chronic cardenolide toxicity are more numerous, and can be subdivided in studies that observed cardenolide effects on growth of individual insects and populations (reviewed in Agrawal et al., 2012), and on insect survival, the fitness component we focused on in our CAFE assays as well. Survival of the non-adapted herbivores fall armyworm (Spodoptera frugiperda) and velvetbean caterpillar (Anticarsia gemmatalis) was affected by digitoxin (Cohen, 1983), whereas survival of the specialist monarch butterfly and milkweed beetle Tetraopes tetraophthalmus was negatively correlated with milkweed cardenolide concentrations (Rasmann et al., 2011; Rasmann and Agrawal, 2011; Zalucki et al., 1990; Zalucki \& Brower, 1992). None of the studies on specialized and non- 
423 adapted herbivorous insects established LD50 values, but the LD50 for the three 424 cardenolides in Drosophila was within the range of LD50 values for these compounds in mammals that are not specialized on cardenolide-containing diets either (Table 1).

Several previous studies on specialized and non-adapted mammals, herbivorous insects and their predators noted feeding deterrent effects of the apolar digitoxin (Akhtar and Isman, 2004; Glendinning, 1992). Digitoxin is reportedly bitterer in taste to mammals than the polar ouabain (Malcolm, 1991), although it is unknown to which extent this is the case for insects. One study reported deterrence by an analogue of ouabain (or g-strophantin), k-strophantin, in the generalist herbivore Helicoverpa armigera (Zhou et al., 2010). Yet, not all studies observed a feeding deterrent effect of either digitoxin or ouabain (Green et al., 2011; Vickerman \& de Boer, 2002), and $D$. melanogaster was not deterred from feeding by cardenolides in our experiments either. What is unclear is whether any of the non-responsive insects are able to perceive the cardenolides via gustatory receptors. biologically relevant cardenolide levels than wildtype flies. Knockout mutants also

Mdr and Oatp knockout mutant flies succumbed more rapidly on diets with experienced more adverse acute neurological effects on diets with higher cardenolide levels. These findings further support the hypothesis that Mdrs and Oatps are important for full protection against dietary cardenolides.

Previous studies of Drosophila have shown that knocking out one transporter is accompanied by the downregulation of the Malpighian tubule-based expression of one or more other transporters (Chahine et al., 2012a and -b). This makes it very difficult to link an effect on lifespan or stress-sensitivity with the contribution of any single transporter. Studies in vertebrates and invertebrates repeatedly emphasize the importance of multiple transporters with overlapping substrate specificities in the response of epithelial tissues to organic toxins (e.g. Wright and Dantzler, 2004). With this caveat in mind, our results suggest that of the Mdrs, the BBB-expressed Mdr65 made the largest contribution in 
450

451

452

453 454

455

456

457

458

459

460

461

462

463

464

465

466

467

468

469

470

471

472

473

474

475

476

protection against cardenolides (Figures 4 and 5). And although redundancy between the midgut-expressed Mdr49 and -50 is possible, the PMdr49 mutant was more sensitive to biologically relevant levels of digitoxin (Figure 4B). Mdr49 is also highly and moderately expressed in the tubules and BBB, respectively (Supplemental Figure 2; DeSalvo et al., 2014). Of the Oatps, Oatp30B appeared to be the most important transporter in resistance to biologically relevant levels of ouabain, and this transporter is moderately expressed in all tissues including the BBB, midgut and tubules (Supplemental Figure 4). Whereas the role of the other two Oatps in protecting flies seemed less important at biologically relevant ouabain levels, both the POatp33Eb and $-58 \mathrm{Db}$ knockout mutants were more sensitive to mechanical stress when fed high levels of ouabain (Figure 7). These Oatps are mainly expressed in the tubules and were previously found to be the most efficient ouabain transporters of all Drosophila Oatps (Torrie et al., 2004).

Not much is known about the importance of Mdrs and Oatps in response to other plant-produced toxins. Virtually nothing is known about Oatps in this context, and only scant evidence exists for Mdrs (Dermauw and Van Leeuwen, 2014). In the generalist herbivore $H$. armigera, flavonoids such as quercetin inhibit Mdr ATPase activity and bind to Mdrs with high affinity (Aurade et al., 2011). In the tobacco specialist Manduca sexta, BBB-expressed Mdrs are involved in the excretion of the alkaloid nicotine (Gaertner et al., 1998; Murray et al., 1994), and Mdr expression is downregulated in larvae feeding on plants with lower nicotine levels (Govind et al., 2010). Finally, although it is difficult to assign a rate-limiting role in transport of a specific organic toxin to a single membrane transporter based on expression data alone (Chahine et al., 2012a and -b), Mdrs seem to be involved in transport of colchicine, a toxin from autumn crocus (Colchicum autumnale): expression of Mdr49 is upregulated in D. melanogaster upon colchicine ingestion and PMdr49 knockout mutants are more sensitive to this toxin (Tapadia and Lakhotia, 2005; Wu et al., 1991). To which extent transporter-mediated protection coincides and interacts with other mechanisms of protection such as 
477 detoxification and target site insensitivity is currently unknown, but the insect adaptations

478 to host plant-produced cardenolides form a powerful model system.

479 Our results support the hypothesis proposed by Dobler and colleagues (2015) that

480 all insects are likely to have at least some level of resistance to cardenolides through the 481 action of the highly conserved Mdr and Oatp transporters. This basal level of resistance 482 might have increased further in species adapted to feeding on cardenolide-producing 483 plants. Physiological evidence supporting this idea has been found in several moth 484 species (Petschenka et al., 2013), and the finding that Na/K-ATPase activity in BBB485 protected neural tissue of the milkweed bug is less sensitive to cardenolides than activity 486 in cell cultures expressing cardenolide-resistant $\mathrm{Na} / \mathrm{K}$-ATPase alleles also points to a 487 protective effect of transporters in specialists (Dalla and Dobler, 2016). Our study 488 provides a foundation for testing this evolutionary scenario via comparative functional 489 genomics (Groen and Whiteman, 2016). Mdrs and Oatps from non-adapted insects and 490 from congeners that are adapted to feeding on cardenolide-containing plants can be 491 expressed heterologously in Drosophila, after which the effects on cardenolide toxicity 492 can be tested in the high-precision CAFE assays. Given our findings that cardenolide 493 LD50 values for Drosophila are comparable to those for vertebrates, this also enhances 494 the prospect of using Drosophila for in vivo functional genetic pharmacological and 495 toxicological tests with cardenolides and possibly other plant secondary metabolites to 496 minimize more expensive and cumbersome tests on vertebrates.

498 Acknowledgements

We thank Tobias Züst for verifying cardenolide concentrations in Drosophila food 501 solutions, Julianne Ray and Joseph Hernandez for assistance with CAFE assays, and 502 Jennifer Koop and Andrew Gloss for advice on assay design and fly stock maintenance. 
503 We are grateful to Safaa Dalla, Amy Hastings, Jennifer Lohr, Georg Petschenka, Sophie 504 Zaaijer, Tobias Züst, and members of the Dobler and Whiteman laboratories for useful 505 discussions, and to Sophie Zaaijer for providing images. Stocks obtained from the 506 Bloomington Drosophila Stock Center (NIH P40OD018537) and the Exelixis Collection

507 at the Harvard Medical School were used in this study. This work was supported by the 508 John Templeton Foundation (SCG, ERL, AAA, SD and NKW; Grant ID \#41855 to 509 AAA, SD, and NKW). SD was supported by the German Science Foundation (DFG, 510 grant Do527/5-1 to SD). NKW was funded by the National Geographic Society (Grant 511 9097-12 to NKW), the University of Arizona (Faculty Seed Grant, Center for Insect 512 Science Seed Grant, and laboratory set-up grant to NKW), the National Science 513 Foundation (grant DEB-1256758 to NKW) and the National Institute of General Medical 514 Sciences of the National Institutes of Health under Award Number R35GM119816. The 515 content is solely the responsibility of the authors and does not necessarily represent the 516 official views of the National Institutes of Health. The funding sources had no 517 involvement in the study design; the collection, analysis and interpretation of data; the 518 writing of the report; or in the decision to submit the article for publication. Data 519 accessibility: all raw data will be deposited in the Dryad data repository (doi \# pending).

\section{References}

523 Agrawal, A.A., Patrick, E.T., Hastings A.P., 2014. Tests of the coupled expression of 524 latex and cardenolide plant defense in common milkweed (Asclepias syriaca). Ecosphere $5255,126$.

526 Agrawal, A.A., Petschenka, G., Bingham, R.A., Weber, M.G., Rasmann, S., 2012. Toxic 527 cardenolides: chemical ecology and coevolution of specialized plant-herbivore 528 interactions. New Phytol. 194, 28-45. 
529 Akhtar, Y., Isman, M.B., 2004. Comparative growth inhibitory and antifeedant effects of 530 plant extracts and pure allelochemicals on four phytophagous insect species. J. Appl. 531 Entomol. 128, 32-38.

532 Ambrosy, A.P., Butler, J., Ahmed, A., Vaduganathan, M., van Veldhuisen, D.J., Colucci, 533 W.S., Gheorghiade, M., 2014. The use of digoxin in patients with worsening chronic 534 heart failure: reconsidering an old drug to reduce hospital admissions. J. Am. Coll. 535 Cardiol. 63, 1823-1832.

536 Armstrong, G.A., Xiao, C., Krill, J.L., Seroude, L., Dawson-Scully, K., Robertson, R.M., 537 2011. Glial Hsp70 protects $\mathrm{K}^{+}$homeostasis in the Drosophila brain during repetitive 538 anoxic depolarization. PLoS ONE 6, e28994.

539 Ashmore, L.J., Hrizo, S.L., Paul, S.M., Van Voorhies, W.A., Beitel, G.J., Palladino, M.J., 540 2009. Novel mutations affecting the $\mathrm{Na}, \mathrm{K}$ ATPase alpha model complex neurological 541 diseases and implicate the sodium pump in increased longevity. Hum. Genet. 126, 431542447.

543 Aurade, R.M., Akbar, S.M., Goud, H., Jayalakshmi, S.K., Sreeramulu, K., 2011.

544 Inhibition of P-glycoprotein ATPase and its transport function of Helicoverpa armigera

545 by morin, quercetin and phloroglucinol. Pestic. Biochem. Physiol. 101, 212-219.

546 Barbehenn, R.V., 1999. Non-absorption of ingested lipophilic and amphiphilic 547 allelochemicals by generalist grasshoppers: the role of extractive ultrafiltration by the 548 peritrophic envelope. Arch. Insect Biochem. Physiol. 42, 130-137.

549 Barbehenn, R.V., 2001. Roles of peritrophic membranes in protecting herbivorous insects 550 from ingested plant allelochemicals. Arch. Insect Biochem. Physiol. 47, 86-99.

551 Beikirch, H., 1977. Toxicity of ouabain on Drosophila melanogaster. Experientia 33, 552 494-495. 
553 Bellen, H.J., Levis, R.W., He, Y., Carlson, J.W., Evans-Holm, M., Bae, E., Kim, J., 554 Metaxakis, A., Savakis, C., Schulze, K.L., Hoskins, R.A, Spradling, A.C., 2011. The 555 Drosophila gene disruption project: progress using transposons with distinctive site 556 specificities. Genetics 188, 731-743.

557 Bellen, H.J., Levis, R.W., Liao, G., He, Y., Carlson, J.W., Tsang, G., Evans-Holm, M., 558 Hiesinger, P.R., Schulze, K.L., Rubin, G.M., Hoskins, R.A., Spradling, A.C., 2004. The 559 BDGP gene disruption project: single transposon insertions associated with $40 \%$ of 560 Drosophila genes. Genetics 167, 761-781.

561 Borst, P., Schinkel, A.H., 2013. P-glycoprotein ABCB1: a major player in drug handling 562 by mammals. J. Clin. Invest. 123, 4131-4133.

563 Brown, J.B., Boley, N., Eisman, R., May, G.E., Stoiber, M.H., Duff, M.O., Booth, B.W., 564 Wen, J., Park, S., Suzuki, A.M., Wan, K.H., Yu, C., Zhang, D., Carlson, J.W., Cherbas, 565 L., Eads, B.D., Miller, D., Mockaitis, K., Roberts, J., Davis, C.A., Frise, E., Hammonds, 566 A.S., Olson, S., Shenker, S., Sturgill, D., Samsonova, A.A., Weiszmann, R., Robinson, 567 G., Hernandez, J., Andrews, J., Bickel, P.J., Carninci, P., Cherbas, P., Gingeras, T.R., 568 Hoskins, R.A., Kaufman, T.C., Lai, E.C., Oliver, B., Perrimon, N., Graveley, B.R., 569 Celniker, S.E., 2014. Diversity and dynamics of the Drosophila transcriptome. Nature $570 \quad 512,393-399$.

571 Chahine, S., Campos, A., O'Donnell, M.J., 2012a. Genetic knockdown of a single organic 572 anion transporter alters the expression of functionally related genes in Malpighian tubules 573 of Drosophila melanogaster. J. Exp. Biol. 215, 2601-2610.

574 Chahine, S., O'Donnell, M.J., 2009. Physiological and molecular characterization of 575 methotrexate transport by Malpighian tubules of adult Drosophila melanogaster. J. Insect 576 Physiol. 55, 927-935.

577 Chahine, S., O'Donnell, M.J., 2011. Interactions between detoxification mechanisms and 578 excretion in Malpighian tubules of Drosophila melanogaster. J. Exp. Biol. 214, 462-468. 
579 Chahine, S., Seabrooke, S., O'Donnell, M.J., 2012b. Effects of genetic knock-down of 580 organic anion transporter genes on secretion of fluorescent organic ions by Malpighian 581 tubules of Drosophila melanogaster. Arch. Insect Biochem. Physiol. 81, 228-240.

582 Cohen, J.A., 1983. Chemical interactions among milkweed plants (Asclepiadaceae) and 583 lepidopteran herbivores. PhD dissertation. University of Florida, Gainesville, FL, USA.

584 Dalla, S., Dobler, S., 2016. Gene duplications circumvent trade-offs in enzyme function: 585 insect adaptation to toxic host plants. Evolution, doi: 10.1111/evo.13077.

586 Dalla, S., Swarts, H.G., Koenderink, J.B., Dobler, S., 2013. Amino acid substitutions of $587 \mathrm{Na}, \mathrm{K}$-ATPase conferring decreased sensitivity to cardenolides in insects compared to 588 mammals. Insect Biochem. Mol. Biol. 43, 1109-1115.

589 Dean, M., Rzhetsky, A., Allikmets, R., 2001. The human ATP-binding cassette (ABC) 590 transporter superfamily. Genome Res. 11, 1156-1166.

591 Dermauw, W., Van Leeuwen, T., 2014. The ABC gene family in arthropods: comparative 592 genomics and role in insecticide transport and resistance. Insect Biochem. Mol. Biol. 45, 593 89-110.

594 DeSalvo, M.K., Hindle, S.J., Rusan, Z.M., Orng, S., Eddison, M., Halliwill, K., Bainton, 595 R.J., 2014. The Drosophila surface glia transcriptome: evolutionary conserved blood596 brain barrier processes. Front. Neurosci. 8, 346.

597 Deshpande, S.A., Carvalho, G.B., Amador, A., Phillips, A.M, Hoxha, S., Lizotte, K.J., Ja, 598 W.W., 2014. Quantifying Drosophila food intake: comparative analysis of current 599 methodology. Nat. Methods 11, 535-540.

600 Dobler, S., Dalla, S., Wagschal, V., Agrawal, A.A., 2012. Community-wide convergent 601 evolution in insect adaptation to toxic cardenolides by substitutions in the Na,K-ATPase. 602 Proc. Natl. Acad. Sci. U.S.A. 109, 13040-13045. 
603 Dobler, S., Petschenka, G., Wagschal, V., Flacht, L., 2015. Convergent adaptive 604 evolution - how insects master the challenge of cardiac glycoside-containing host plants. 605 Ent. Exp. Appl. 157, 30-39.

606 Dow, J.A., Davies, S.A., 2006. The Malpighian tubule: rapid insights from post-genomic 607 biology. J. Insect Physiol. 52, 365-378.

608 Dussourd, D.E., Hoyle, A.M., 2000. Poisoned plusiines: toxicity of milkweed latex and 609 cardenolides to some generalist caterpillars. Chemoecology 10, 11-16.

610 Frick, C., Wink, M., 1995. Uptake and sequestration of ouabain and other cardiac 611 glycosides in Danaus plexippus (Lepidoptera: Danaidae): evidence for a carrier-mediated 612 process. J. Chem. Ecol. 21, 557-575.

613 Gaertner, L.S., Murray, C.L., Morris, C.E., 1998. Transepithelial transport of nicotine and 614 vinblastine in isolated Malpighian tubules of the tobacco hornworm (Manduca sexta) 615 suggests a P-glycoprotein-like mechanism. J. Exp. Biol. 201, 2637-2645.

616 Ganetzky, B., Wu, C., 1982. Indirect suppression involving mutants with altered nerve 617 excitability in Drosophila melanogaster. Genetics 100, 597-614.

618 Gerrard, B., Stewart, C., Dean, M., 1993. Analysis of Mdr50: a Drosophila P619 glycoprotein/multidrug resistance gene homolog. Genomics 17, 83-88.

620 Glendinning, J.I., 1992. Effectiveness of cardenolides as feeding deterrents to 621 Peromyscus mice. Journal of Chemical Ecology 18, 1559-1575.

622 Govind, G., Mittapalli, O., Griebel, T., Allmann, S., Bocker, S., Baldwin, I.T., 2010. 623 Unbiased transcriptional comparisons of generalist and specialist herbivores feeding on 624 progressively defenseless Nicotiana attenuata plants. PLoS ONE 5, e8735. 
625 Gozalpour, E., Wittgen, H.G.M., van den Heuvel, J.J.M.W., Greupink, R., Russel, 626 F.G.M., Koenderink, J.B., 2013. Interaction of digitalis-like compounds with P627 glycoprotein. Toxicol. Sci. 131, 502-511.

628 Graveley, B.R., Brooks, A.N., Carlson, J.W., Duff, M.O., Landolin, J.M., Yang, L., 629 Artieri, C.G., van Baren, M.J., Boley, N., Booth, B.W., Brown, J.B., Cherbas, L., Davis, 630 C.A., Dobin, A., Li, R., Lin, W., Malone, J.H., Mattiuzzo, N.R., Miller, D., Sturgill, D., 631 Tuch, B.B., Zaleski, C., Zhang, D., Blanchette, M., Dudoit, S., Eads, B., Green, R.E., 632 Hammonds, A., Jiang, L., Kapranov, P., Langton, L., Perrimon, N., Sandler, J.E., Wan, 633 K.H., Willingham, A., Zhang, Y., Zou, Y., Andrews, J., Bickel, P.J., Brenner, S.E., Brent, 634 M.R., Cherbas, P., Gingeras, T.R., Hoskins, R.A., Kaufman, T.C., Oliver, B., Celniker, 635 S.E., 2011. The developmental transcriptome of Drosophila melanogaster. Nature 471, $636 \quad 473-479$.

637 Green, P., Veitch, N., Stevenson, P., Simmonds, M., 2011. Cardenolides from 638 Gomphocarpus sinaicus and Pergularia tomentosa (Apocynaceae: Asclepiadoideae) 639 deter the feeding of Spodoptera littoralis. Arthropod-Plant Interactions 5, 219-225.

640 Groen, S.C., Whiteman, N.K., 2016. Using Drosophila to study the evolution of 641 herbivory and diet specialization. Curr. Opin. Insect Sci. 14, 66-72.

642 Hagenbuch, B., Stieger, B., 2013. The SLCO (former SLC21) superfamily of 643 transporters. Mol. Aspects Med. 34, 396-412.

644 Hindle, S.J., Bainton, R.J., 2014. Barrier mechanisms in the Drosophila blood-brain 645 barrier. Front. Neurosci. 8, 414.

646 Huang, H., Lu-Bo, Y., Haddad, G.G., 2014. A Drosophila ABC transporter regulates 647 lifespan. PLoS Genet. 10, e1004844. 
648 Ja, W.W., Carvalho, G.B., Mak, E.M., de la Rosa, N.N., Fang, A.Y., Liong, J.C., 649 Brummel, T., Benzer, S., 2007. Prandiology of Drosophila and the CAFE assay. Proc. 650 Natl. Acad. Sci. U.S.A. 104, 8253-8256.

651 Kearney, J.N., 1983. Selection and utilization of natural substrates as breeding sites by 652 woodland Drosophila spp. Ent. Exp. Appl. 33, 63-70.

653 Lee, K.P., Simpson, S.J., Clissold, F.J., Brooks, R., Ballard, J.W.O., Taylor, P.W., Soran, 654 N., Raubenheimer, D., 2008. Lifespan and reproduction in Drosophila: new insights from 655 nutritional geometry. Proc. Natl. Acad. Sci. U.S.A. 105, 2498-2503.

656 Malcolm, S., 1991. Cardenolide-mediated interactions between plants and herbivores, in: 657 Rosenthal, G.A., Berenbaum, M.R. (Eds.), Herbivores, Their Interaction with Secondary 658 Plant Metabolites. Academic Press Inc, New York, pp. 251-296.

659 Marty, M.A., Krieger, R.I., 1984. Metabolism of uscharidin, a milkweed cardenolide, by 660 tissue homogenates of monarch butterfly larvae, Danaus plexippus L. J. Chem. Ecol. 10, $661945-956$.

662 Mayer, F., Mayer, N., Chinn, L., Pinsonneault, R.L., Kroetz, D., Bainton, R.J., 2009. 663 Evolutionary conservation of vertebrate blood-brain barrier chemoprotective mechanisms 664 in Drosophila. J. Neurosci. 29, 3538-3550.

665 Meredith, J., Moore, L., Scudder, G.G.E., 1984. Excretion of ouabain by Malpighian 666 tubules of Oncopeltus fasciatus. Am. J. Physiol. 246, R705-R715.

667 Murray, C.L., Quaglia, M., Arnason, J.T., Morris, C.E., 1994. A putative nicotine pump 668 at the metabolic blood-brain-barrier of the tobacco hornworm. J. Neurobiol. 25, 23-34.

669 Nazir, A., Mukhopadhyay, I., Saxena, D.K., Kar Chowdhuri, D., 2003. Evaluation of no 670 observed adverse effect level (NOAEL) of solvent dimethyl sulphoxide in Drosophila 671 melanogaster: a developmental, reproductive and cytotoxicity study. Toxicol. Mech. 672 Methods 13, 147-152. 
673 Obaidat, A., Roth, M., Hagenbuch, B., 2012. The expression and function of organic 674 anion transporting polypeptides in normal tissues and in cancer. Annu. Rev. Pharmacol. 675 Toxicol. 52, 135-151.

676 Oxenkrug, G.F., 2010. The extended life span of Drosophila melanogaster eye-color 677 (white and vermilion) mutants with impaired formation of kynurenine. J. Neural Transm. $678117,23-26$.

679 Parks, A.L., Cook, K.R., Belvin, M., Dompe, N.A., Fawcett, R., Huppert, K., Tan, L.R., 680 Winter, C.G., Bogart, K.P., Deal, J.E., Deal-Herr, M.E., Grant, D., Marcinko, M., 681 Miyazaki, W.Y., Robertson, S., Shaw, K.J., Tabios, M., Vysotskaia, V., Zhao, L., 682 Andrade, R.S., Edgar, K.A., Howie, E., Killpack, K., Milash, B., Norton, A., Thao, D., 683 Whittaker, K., Winner, M.A., Friedman, L., Margolis, J., Singer, M.A., Kopczynski, C., 684 Curtis, D., Kaufman, T.C., Plowman, G.D., Duyk, G., Francis-Lang, H.L., 2004. 685 Systematic generation of high-resolution deletion coverage of the Drosophila 686 melanogaster genome. Nat. Genet. 36, 288-292.

687 Pegueroles, C., Ferrés-Coy, A., Martí-Solano, M., Aquadro, C.F., Pascual, M., Mestres, 688 F., 2016. Inversions and adaptation to the plant toxin ouabain shape DNA sequence 689 variation within and between chromosomal inversions of Drosophila subobscura. Sci. 690 Rep. 6, 23754.

691 Petschenka, G., Agrawal, A.A., 2015. Milkweed butterfly resistance to plant toxins is 692 linked to sequestration, not coping with a toxic diet. Proc. Biol. Sci. 282, 20151865.

693 Petschenka, G., Agrawal, A.A., 2016. How herbivores coopt plant defenses: natural 694 selection, specialization, and sequestration. Curr. Opin. Insect Sci. 14, 17-24.

695 Petschenka, G., Dobler, S., 2009. Target-site sensitivity in a specialized herbivore 696 towards major toxic compounds of its host plant: the $\mathrm{Na}^{+} \mathrm{K}^{+}$-ATPase of the oleander 697 hawk moth (Daphnis nerii) is highly susceptible to cardenolides. Chemoecology 19, 235698239. 
699 Petschenka, G., Offe, J.K., Dobler, S., 2012. Physiological screening for target site 700 insensitivity and localization of $\mathrm{Na}^{+} / \mathrm{K}^{+}$-ATPase in cardenolide-adapted Lepidoptera. $\mathrm{J}$. 701 Insect Physiol. 58, 607-612.

702 Petschenka, G., Pick, C., Wagschal, V., Dobler, S., 2013. Functional evidence for 703 physiological mechanisms to circumvent neurotoxicity of cardenolides in an adapted and 704 a non-adapted hawk-moth species. Proc. Biol. Sci. 280, 20123089.

705 R Core Development Team, 2012. R: a language and environment for statistical 706 computing.

707 Rasmann, S., Agrawal, A.A., 2011. Evolution of specialization: a phylogenetic study of 708 host range in the red milkweed beetle (Tetraopes tetraophthalmus). American Naturalist $709 \quad 177,728-737$.

710 Rasmann, S., Erwin, A.C., Halitschke, R., Agrawal, A.A., 2011. Direct and indirect root 711 defense of milkweed (Asclepias syriaca): trophic cascades, tradeoffs, and novel methods 712 for studying subterranean herbivory. Journal of Ecology 99, 16-25.

713 Rasmann, S., Johnson, M.D., Agrawal, A.A., 2009. Induced responses to herbivory and 714 jasmonate in three milkweed species. J. Chem. Ecol. 35, 1326-1334.

715 Sachdev-Gupta, K., Radke, C., Renwick, J.A., Dimock, M.B., 1993. Cardenolides from 716 Erysimum cheiranthoides: feeding deterrents to Pieris rapae larvae. J. Chem. Ecol. 19, $717 \quad 1355-1369$.

718 dos Santos, G., Schroeder, A.J., Goodman, J.L., Strelets, V.B., Crosby, M.A., Thurmond, 719 J., Emmert, D.B., Gelbart, W.M., FlyBase Consortium, 2015. FlyBase: introduction of 720 the Drosophila melanogaster Release 6 reference genome assembly and large-scale 721 migration of genome annotations. Nucleic Acids Res. 43, D690-D697. 
722 Schubiger, M., Feng, Y., Fambrough, D.M., Palka, J., 1994. A mutation of the 723 Drosophila sodium pump alpha subunit gene results in bang-sensitive paralysis. Neuron $724 \quad 12,373-381$.

725 Scudder, G.G.E., Meredith, J., 1982. The permeability of the midgut of three insects to 726 cardiac glycosides. J. Insect Physiol. 28, 689-694.

727 Seabrooke, S., O'Donnell, M.J., 2013. Oatp58Dc contributes to blood-brain barrier 728 function by excluding organic anions from the Drosophila brain. Am. J. Physiol. Cell. 729 Physiol. 305, C558-C567.

730 Seiber, J.N., Tuskes, P.M., Brower, L.P., Nelson, C.J., 1980. Pharmacodynamics of some 731 individual milkweed cardenolides fed to larvae of the monarch butterfly (Danaus 732 plexippus L.). J. Chem. Ecol. 6, 321-339.

733 Stern, D.L.,2013. The genetic causes of convergent evolution. Nat. Rev. Genet. 14, 751734764.

735 Storz, J.F., 2016. Causes of molecular convergence and parallelism in protein evolution. 736 Nat. Rev. Genet. 17, 239-250.

737 Tapadia, M.G., Lakhotia, S.C., 2005. Expression of Mdr49 and Mdr65 multidrug 738 resistance genes in larval tissues of Drosophila melanogaster under normal and stress 739 conditions. Cell Stress Chaperones 10, 7-11.

740 Thibault, S.T., Singer, M.A., Miyazaki, W.Y., Milash, B., Dompe, N.A., Singh, C.M., 741 Buchholz, R., Demsky, M., Fawcett, R., Francis-Lang, H.L., Ryner, L., Cheung, L.M., 742 Chong, A., Erickson, C., Fisher, W.W., Greer, K., Hartouni, S.R., Howie, E., Jakkula, L., 743 Joo, D., Killpack, K., Laufer, A., Mazzotta, J., Smith, R.D., Stevens, L.M., Stuber, C., 744 Tan, L.R., Ventura, R., Woo, A., Zakrajsek, I., Zhao, L., Chen, F., Swimmer, C., 745 Kopczynski, C., Duyk, G., Winberg, M.L., Margolis, J., 2004. A complementary 
746 transposon tool kit for Drosophila melanogaster using $P$ and piggyBac. Nat. Genet. 36, $747 \quad 283-287$.

748 Torrie, L.S., Radford, J.C., Southall, T.D., Kean, L., Dinsmore, A.J., Davies, S.A., Dow, 749 J.A., 2004. Resolution of the insect ouabain paradox. Proc. Natl. Acad. Sci. U.S.A. 101, $750 \quad 13689-13693$.

751 Vickerman, D.B., de Boer, G., 2002. Maintenance of narrow diet breadth in the monarch

752 butterfly caterpillar: response to various plant species and chemicals. Entomologia

753 Experimentalis et Applicata 104, 255-269.

754 Vigne, P., Frelin, C., 2007. Diet dependent longevity and hypoxic tolerance of adult 755 Drosophila melanogaster. Mech. Age Dev. 128, 401-406.

756 Vigne, P., Frelin, C., 2010. Hypoxia modifies the feeding preferences of Drosophila. 757 Consequences for diet dependent hypoxic survival. BMC Physiol. 10, 8.

758 Wangler, M.F., Yamamoto, S., Bellen, H.J., 2015. Fruit flies in biomedical research. 759 Genetics 199, 639-653.

760 Wright, S.H., Dantzler, W.H., 2004. Molecular and cellular physiology of renal organic 761 cation and anion transport. Physiol. Rev. 84, 987-1049.

762 Wu, C.T., Budding, M., Griffin, M.S., Croop, J.M., 1991. Isolation and characterization 763 of Drosophila multidrug resistance gene homologs. Mol. Cell Biol. 11, 3940-3948.

764 Xia, S.Z., Feng, C.H., Guo, A.K., 1998. Multiple-phase model of memory consolidation 765 confirmed by behavioral and pharmacological analyses of operant conditioning in 766 Drosophila. Pharmacol. Biochem. Behav. 60, 809-816.

767 Xia, S., Liu, L., Feng, C., Guo, A., 1997. Drug disruption of short-term memory in 768 Drosophila melanogaster. Pharmacol. Biochem. Behav. 58, 727-735.

769 Zalucki, M.P., Brower, L.P., 1992. Survival of first instar larvae of Danaus plexippus 
770 (Lepidoptera: Danainae) in relation to cardiac glycoside and latex content of Asclepias

771 humistrata (Asclepiadaceae). Chemoecology 3, 81-93.

772 Zalucki, M.P., Brower, L.P., Malcolm, S.B., 1990. Oviposition by Danaus plexippus in 773 relation to cardenolide content of three Asclepias species in the southeastern USA. 774 Ecological Entomology 15, 231-240.

775 Zhen, Y., Aardema, M.L., Medina, E.M., Schumer, M., Andolfatto, P., 2012. Parallel 776 molecular evolution in an herbivore community. Science 337, 1634-1647.

777 Zhou, D., van Loon, J.J., Wang, C.Z., 2010. Experience-based behavioral and 778 chemosensory changes in the generalist insect herbivore Helicoverpa armigera exposed 779 to two deterrent plant chemicals. J. Comp. Physiol. A Neuroethol. Sens. Neural Behav. 780 Physiol. 196, 791-799.

781 Züst, T., Agrawal, A.A., 2016. Population growth and sequestration of plant toxins along 782 a gradient of specialization in four aphid species on the common milkweed Asclepias 783 syriaca. Func. Ecol. 30, 547-556.

784

\section{Tables}

786

787 Table 1. LD50 of cardenolides in Drosophila compared to vertebrate animals.

788

789 Figure Legends

790

791 Figure 1. Cardenolides used in this study. 
793

794

795

796

797

798

799

800

801

802

803

804

805

806

807

808

809

810

811

812

813

814

815

816

817

818

Figure 2. Drosophila shows a non-monotonic dose response to ouabain. (A) Average median lifespan of wildtype (OR) Drosophila melanogaster (Drosophila) males and females feeding on 5\% sucrose (SUC) diets without or with different ouabain (OUA) concentrations. Sex, diet, and their interaction had effects on lifespan (two-way ANOVA, sex: $\mathrm{P}=0.0004$, diet: $\mathrm{P}=2.2 \mathrm{e}-16$, sex $\mathrm{x}$ diet: $\mathrm{P}=0.0132$ ). (B) Average LD50 of wildtype Drosophila males and females expressed as amount of ouabain-containing solution in $\mu \mathrm{L}$ necessary to kill 50\% of flies. Sex, diet, and their interaction had effects on LD50 (twoway ANOVA, sex: $\mathrm{P}=0.0004$, diet: $\mathrm{P}=2.2 \mathrm{e}-16$, sex $\mathrm{x}$ diet: $\mathrm{P}=2.3 \mathrm{e}-12$ ). Letters indicate statistically significant differences between males feeding on different diets (blue capital letters) and, separately, indicate statistically significant differences between females feeding on different diets (red bold letters) (one-way ANOVA with post hoc Tukey's HSD tests, $\mathrm{P}<0.05)$. Data points labeled with the same letter are not significantly different. $\mathrm{N}=4$ vials with 3 flies each per treatment group.

Figure 3. Polar and non-polar cardenolides are toxic to Drosophila. (A) Average feeding rate of wildtype (OR) Drosophila melanogaster (Drosophila) males and females during the first day of feeding on $5 \%$ sucrose (SUC) diets without or with $0.5 \mu \mathrm{M}$ ouabain (OUA), digoxin (DIG) or digitoxin (DGT). Sex, but neither diet nor the sex $\mathrm{x}$ diet interaction, had effects on feeding rate (two-way ANOVA, sex: $\mathrm{P}=0.0030$, diet: $\mathrm{P}=$ 0.0678, sex x diet: $\mathrm{P}=0.6729$ ). (B) Average median lifespan of wildtype Drosophila males and females feeding on $5 \%$ sucrose diets without or with different cardenolides. Diet and sex, but not the sex $\mathrm{x}$ diet interaction, had effects on lifespan (two-way ANOVA, sex: $\mathrm{P}=0.0335$, diet: $\mathrm{P}=9.743 \mathrm{e}-8$, sex $\mathrm{x}$ diet: $\mathrm{P}=0.2812$ ). (C) Average LD50 of wildtype Drosophila males and females expressed as amount of cardenolide in ng necessary to kill $50 \%$ of flies. Sex and diet, but not their interaction, had effects on LD50 (two-way ANOVA, sex: $\mathrm{P}=0.0051$, diet: $\mathrm{P}=1.337 \mathrm{e}-7$, sex $\mathrm{x}$ diet: $\mathrm{P}=0.3937$ ). (D) 
Average LD50 of wildtype Drosophila males and females expressed as amount of cardenolide-containing solution in $\mu \mathrm{L}$ necessary to kill $50 \%$ of flies. Sex and diet, but not their interaction, had effects on LD50 (two-way ANOVA, sex: $\mathrm{P}=0.0034$, diet: $\mathrm{P}=$ 2.562e-10, sex x diet: $\mathrm{P}=0.2697)$. Letters indicate statistically significant differences among groups of males feeding on different diets (bold letters) and among groups of females feeding on different diets (capital letters) (one-way ANOVA with post hoc Tukey's HSD tests, $\mathrm{P}<0.05)$. $\mathrm{N}=4$ vials with 3 flies each per treatment group.

Figure 4. Mdr knockout mutants are relatively more sensitive to cardenolide toxicity than wildtype flies. (A) Average median lifespan of wildtype (OR) and Mdr mutant Drosophila melanogaster (Drosophila) males and females feeding on 5\% sucrose (SUC) diets containing $0.5 \mu \mathrm{M}$ digoxin (DIG) or digitoxin (DGT) relative to the lifespan of these fly genotypes feeding on a control 5\% sucrose diet. Genotype, diet and the genotype $x$ diet interaction, but not sex or any of the interactions that include sex, had effects on lifespan (three-way ANOVA, genotype: $\mathrm{P}=2.374 \mathrm{e}-8$, diet: $\mathrm{P}=3.639 \mathrm{e}-11$, genotype $x$ diet: $\mathrm{P}=9.472 \mathrm{e}-6$, others: $\mathrm{P}>0.05)$. Since sex had no effect, data for males and females were pooled for subsequent tests. Letters indicate statistically significant differences among the fly treatment groups feeding on the digoxin-containing diet (capital letters) and among the fly treatment groups feeding on the digitoxin-containing diet (bold letters) as determined by subsequent analysis on the normalized data (one-way ANOVA with post hoc Tukey's HSD tests, $\mathrm{P}<0.05)$. In the normalization step the median lifespan data for the cardenolide treatment groups were converted so that the data reflect median lifespan relative to the median lifespan of flies in the sucrose control treatment group of the same genotype. $\mathrm{N}=4$ vials with 3 flies each per treatment group. Non-relative data are presented in Supplemental Figure 2. (B) Average LD50 of wildtype and Mdr mutant Drosophila males and females expressed as amount of cardenolide in ng necessary to kill $50 \%$ of flies. Genotype, diet, their interaction, sex and the three-way 
interaction between these factors had significant effects on LD50 (three-way ANOVA, genotype: $\mathrm{P}<2.2 \mathrm{e}-16$, diet: $\mathrm{P}<2.2 \mathrm{e}-16$, genotype $\mathrm{x}$ diet: $\mathrm{P}<2.2 \mathrm{e}-16$, sex: $\mathrm{P}=0.0004$, genotype $\mathrm{x}$ diet $\mathrm{x}$ sex: $\mathrm{P}=0.0192$, others: $\mathrm{P}>0.05)$. Letters indicate statistically significant differences among the groups of male and among the groups of female flies feeding on the digoxin-containing diet (capital letters) and among the groups of male and among the groups of female flies feeding on the digitoxin-containing diet (bold letters) (one-way ANOVA with post hoc Tukey's HSD tests, $\mathrm{P}<0.05$ ). $\mathrm{N}=4$ vials with 3 flies each per treatment group. Results presented for wildtype flies are the same as the results presented for wildtype flies of the same treatment groups in Figure 3.

Figure 5. Mdr knockout mutants show more severe stress-induced paralysis in response to digoxin. (A) Average recovery time after 10s of vortexing of wildtype (OR) and Mdr mutant Drosophila melanogaster (Drosophila) flies feeding on 5\% sucrose (SUC) diets without or with $0.5 \mathrm{mM}$ digoxin (DIG). Genotype, diet and their interaction had effects on recovery time (two-way ANOVA, genotype: $\mathrm{P}=0.0096$, diet: $\mathrm{P}=0.0070$, genotype $\mathrm{x}$ diet: $\mathrm{P}=0.0144)$. Data for males and females were pooled for statistical analysis. Letters indicate statistically significant differences among the fly genotypes feeding on the digoxin-containing diet (capital letters) and among the fly genotypes feeding on the sucrose-containing diet (small letters) (one-way ANOVA with post hoc Tukey's HSD tests, $\mathrm{P}<0.05)$. Letters also reflect statistically significant differences among the fly treatment groups within each genotype (digoxin: capital letters, sucrose: small letters) (Student's t-tests, $\mathrm{P}<0.05$ ). (B) Mortality of wildtype and Mdr mutant Drosophila flies feeding on $5 \%$ sucrose diets without or with $0.5 \mathrm{mM}$ digoxin. Letters indicate statistically significant differences among the fly genotypes feeding on the digoxin-containing diet (capital letters) and among the fly genotypes feeding on the sucrose-containing diet (small letters); letters also reflect statistically significant differences among the fly treatment groups within each genotype (digoxin: capital letters, sucrose: small letters); 
873 the asterisk indicates a larger relative difference between the treatment groups among 874 PMdr65 mutant flies than among wildtype flies (t-tests for binomial proportions, $\mathrm{P}<$ 875 0.05). $\mathrm{N}=37-58$ flies per treatment group.

876

877 Figure 6. Oatp knockout mutants are relatively more sensitive to cardenolide toxicity than 878 wildtype flies. (A) Average median lifespan of wildtype (OR) and Oatp mutant 879 Drosophila melanogaster (Drosophila) males and females feeding on 5\% sucrose (SUC) 880 diets with $0.5 \mu \mathrm{M}$ ouabain (OUA) relative to the lifespan of these fly genotypes feeding 881 on a control 5\% sucrose diet. Genotype, but neither sex nor the genotype x sex 882 interaction, had effects on lifespan (two-way ANOVA, genotype: $\mathrm{P}=0.0049$, sex: $\mathrm{P}=$ 883 0.7571, genotype x sex: $\mathrm{P}=0.9941)$. Since sex had no significant effect, data for males 884 and females were pooled for subsequent tests. Letters indicate statistically significant 885 differences among the groups of flies feeding on the ouabain-containing diet (capital 886 letters) as determined by subsequent analysis on the normalized data (one-way ANOVA 887 with post hoc Tukey's HSD tests, $\mathrm{P}<0.05)$. In the normalization step the median lifespan 888 data for the ouabain treatment group were converted so that the data reflect median 889 lifespan relative to the median lifespan of flies in the sucrose control treatment group of 890 the same genotype. $\mathrm{N}=4$ vials with 3 flies each per treatment group. Non-relative data 891 are presented in Supplemental Figure 4. (B) Average LD50 of wildtype and Oatp mutant 892 Drosophila males and females expressed as amount of ouabain in ng necessary to kill $89350 \%$ of flies. Genotype, but neither sex nor the genotype x sex interaction, had effects on 894 LD50, although the effect of sex did show a trend in that direction (two-way ANOVA, 895 genotype: $\mathrm{P}=2.078 \mathrm{e}-05$, sex: $\mathrm{P}=0.0884$, genotype $\mathrm{x}$ sex: $\mathrm{P}=0.2062$ ). Letters indicate 896 statistically significant differences among the groups of male and among the groups of 897 female flies feeding on the ouabain-containing diet (capital letters) (one-way ANOVA 898 with post hoc Tukey's HSD tests, $\mathrm{P}<0.05) . \mathrm{N}=4$ vials with 3 flies each per treatment 899 group. 
901 Figure 7. Oatp knockout mutants show more severe stress-induced paralysis in response 902 to ouabain. (A) Average recovery time after 10s of vortexing of wildtype (OR) and Oatp 903 mutant Drosophila melanogaster (Drosophila) flies feeding on 5\% sucrose (SUC) diets 904 without or with $0.5 \mathrm{mM}$ or $5 \mathrm{mM}$ ouabain (OUA). Diet, but neither genotype nor the 905 genotype $\mathrm{x}$ diet interaction had significant effects on recovery time (two-way ANOVA, 906 genotype: $\mathrm{P}=0.6450$, diet: $\mathrm{P}=0.0192$, genotype $\mathrm{x}$ diet: $\mathrm{P}=0.3427$ ). Data for males and 907 females were pooled for statistical analysis. There were no differences among the fly 908 genotypes feeding on the ouabain-containing diet or among the fly genotypes feeding on 909 the sucrose-containing diet (one-way ANOVA with post hoc Tukey's HSD tests, P > 910 0.05). Differences among the fly treatment groups within each genotype were not 911 significant either (Student's t-tests, P > 0.05). (B) Mortality of wildtype and Oatp mutant 912 Drosophila flies feeding on $5 \%$ sucrose diets without or with $0.5 \mathrm{mM}$ or $5 \mathrm{mM}$ ouabain. 913 Letters indicate statistically significant differences among the fly genotypes feeding on 914 the ouabain-containing diets (capital letters) and among the fly genotypes feeding on the 915 sucrose-containing diet (small letters). Letters also reflect statistically significant 916 differences among the fly treatment groups within each genotype (ouabain: capital letters, 917 sucrose: small letters). Asterisks indicate a larger relative difference between the 918 treatment groups among POatp58D $\mathrm{b}$ mutant flies than among wildtype flies (t-tests for 919 binomial proportions, $\mathrm{P}<0.05) . \mathrm{N}=20-24$ flies per treatment group. (C) Average 920 recovery time after 10 s of vortexing of wildtype and POatp33Eb mutant Drosophila flies 921 feeding on $5 \%$ sucrose diets without or with $0.5 \mathrm{mM}$ or $5 \mathrm{mM}$ ouabain. Neither genotype 922 nor diet had effects on lifespan (two-way ANOVA, genotype: $\mathrm{P}=0.9960$, diet: $\mathrm{P}=$ 923 0.1458). Data for males and females were pooled for statistical analysis. (D) Mortality of 924 wildtype and POatp33Eb mutant Drosophila flies feeding on 5\% sucrose diets without or 925 with $0.5 \mathrm{mM}$ or $5 \mathrm{mM}$ ouabain. Letters indicate statistically significant differences among 926 the fly genotypes feeding on the ouabain-containing diets (capital letters) and among the 927 fly genotypes feeding on the sucrose-containing diet (small letters); letters also reflect 
928 statistically significant differences among the fly treatment groups within each genotype 929 (ouabain: capital letters, sucrose: small letters); the asterisk indicates a larger relative 930 difference between the treatment groups among POatp33Eb mutant flies than among 931 wildtype flies ( $\mathrm{t}$-tests for binomial proportions, $\mathrm{P}<0.05$ ). $\mathrm{N}=6-8$ flies per treatment 932 group due to a high mortality rate of POatp33Eb flies in culture.

933 

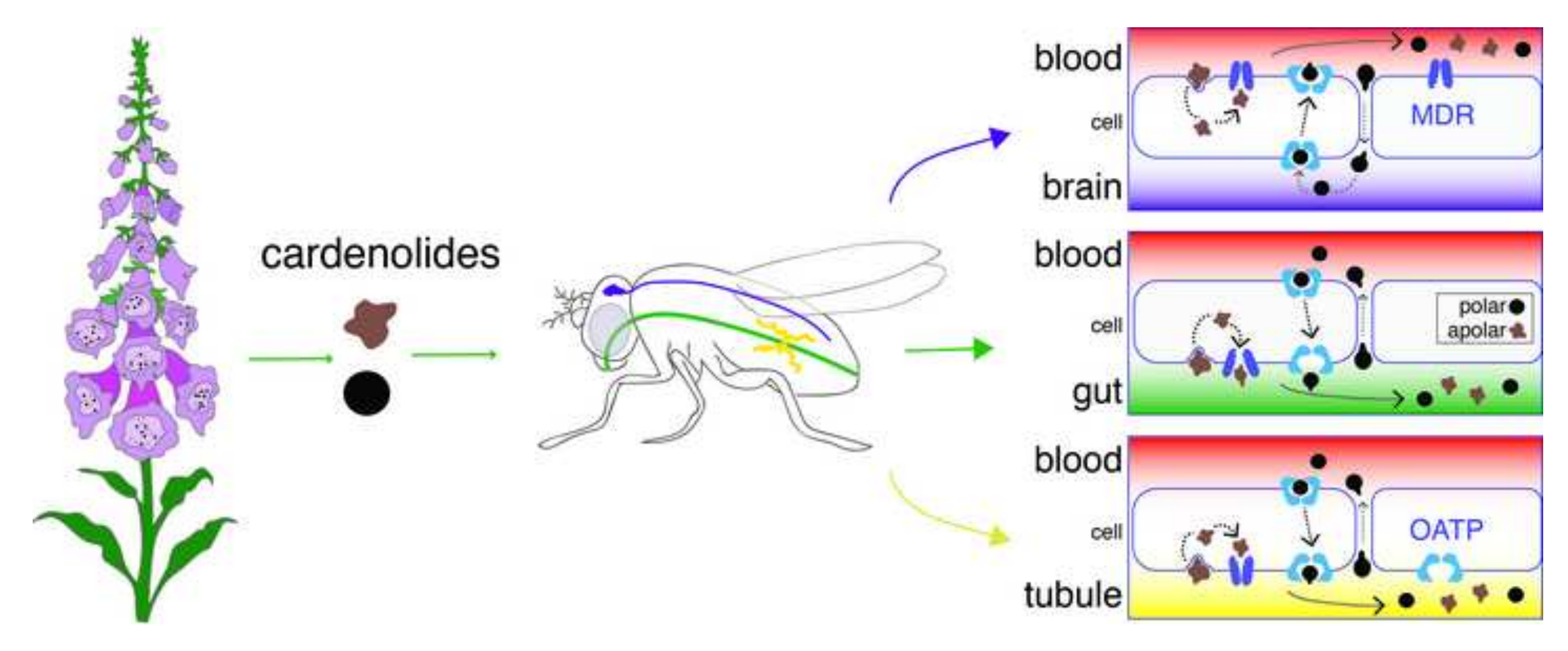

\section{cardenolides}

blood

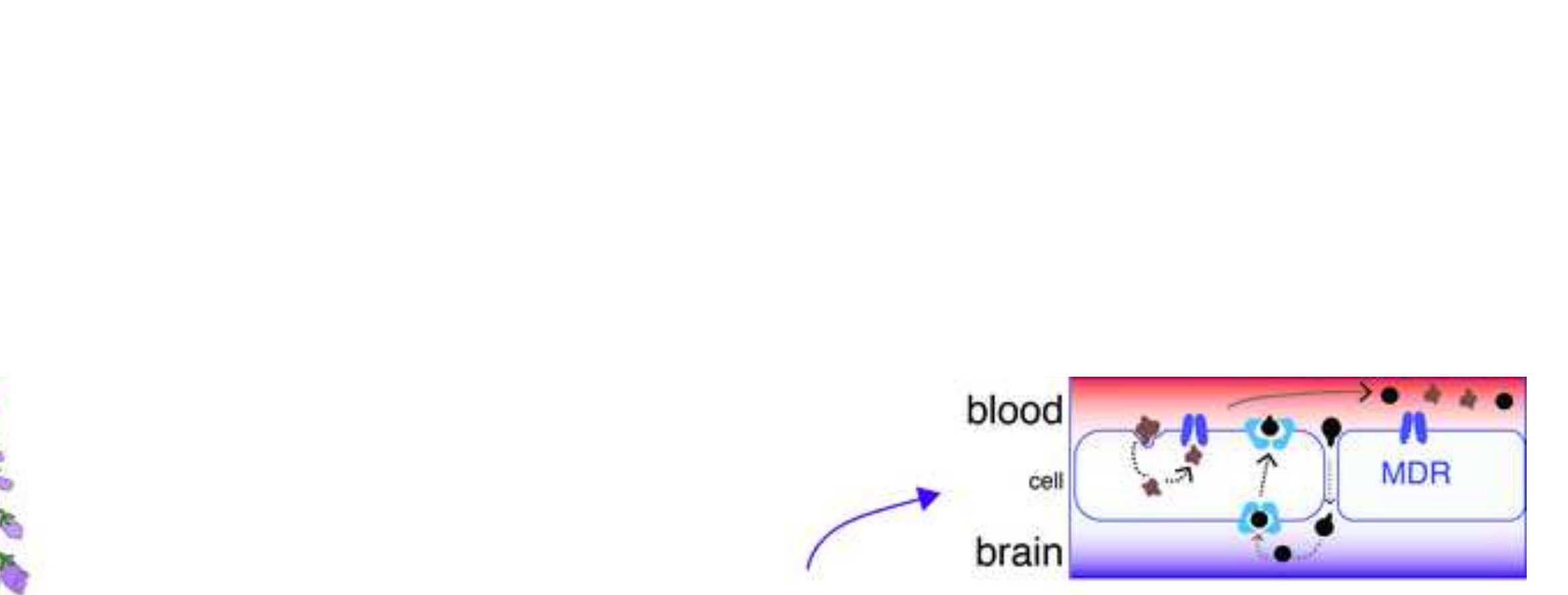


Table 1. LD50 of cardenolides in Drosophila compared to vertebrate animals.

\begin{tabular}{|l|l|l|l|l|l|l|}
\hline \multicolumn{7}{|c|}{ Oral LD50 $(\mathrm{mg} / \mathrm{kg})$} \\
\hline $\begin{array}{l}\text { Cardenolide } \\
\text { Species }\end{array}$ & Drosophila & Frog & Mouse & Rat & Guinea pig & Cat \\
\hline Ouabain & $15.57-16.72$ & & 5 & $3.4-125$ & 8.28 & \\
\hline Digoxin & $17.89-19.45$ & & 17.78 & 28.27 & 3.5 & 0.2 \\
\hline Digitoxin & $13.02-14.92$ & 3.05 & 4.95 & 23.75 & 3.7 & 0.18 \\
\hline
\end{tabular}

The values for Drosophila indicate the ranges of toxicity between male and female animals. The values for ouabain toxicity in rats reflect a range of separate studies. Source: NIH TOXNET ChemIDplus database. 


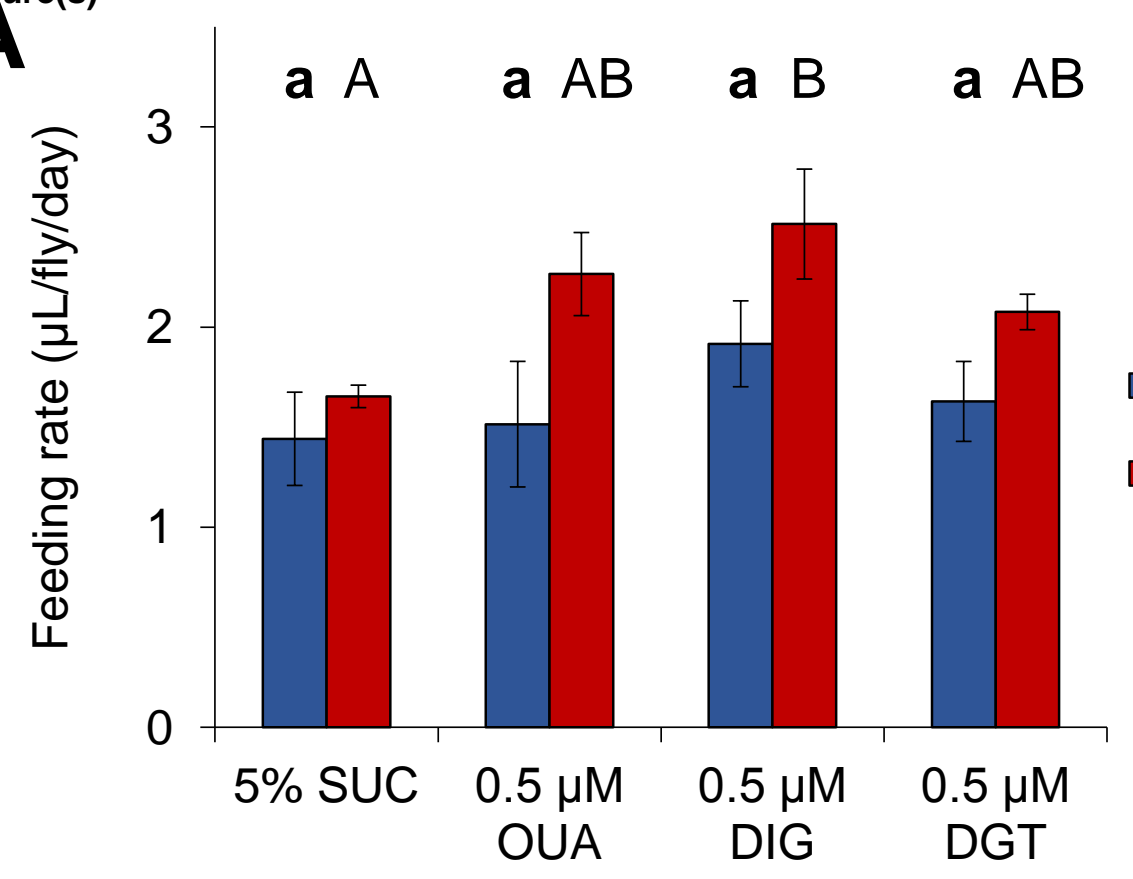

C

- Male

$\square$ Female

b $\mathrm{B}$ c $\mathrm{C}$ a $\mathrm{A}$

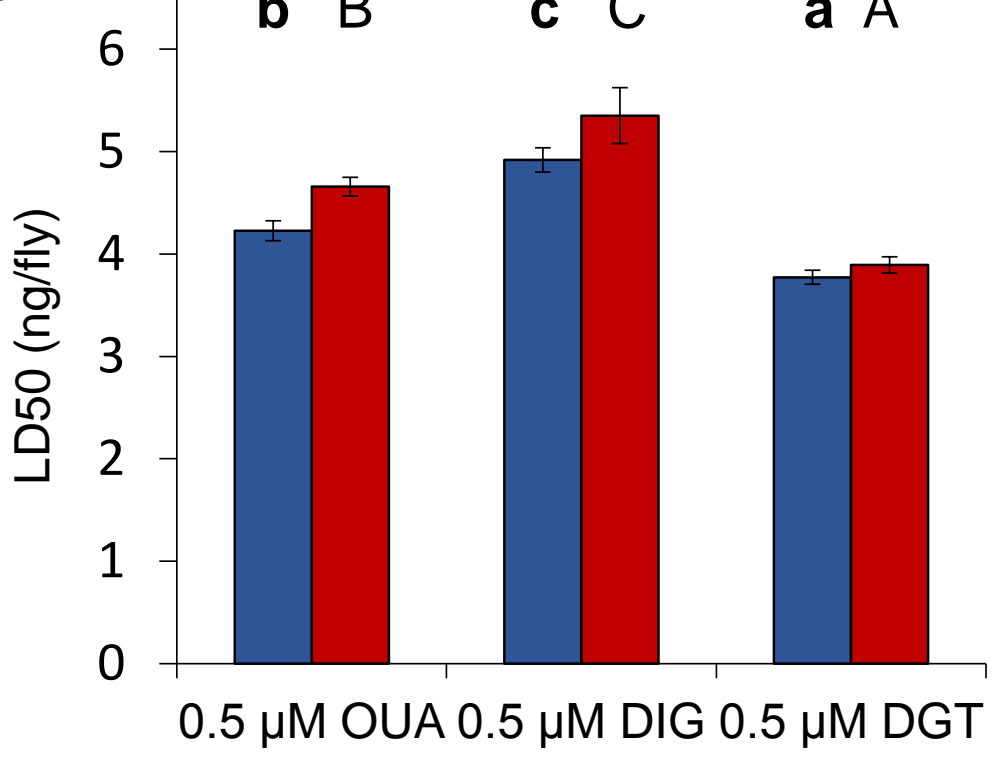

B

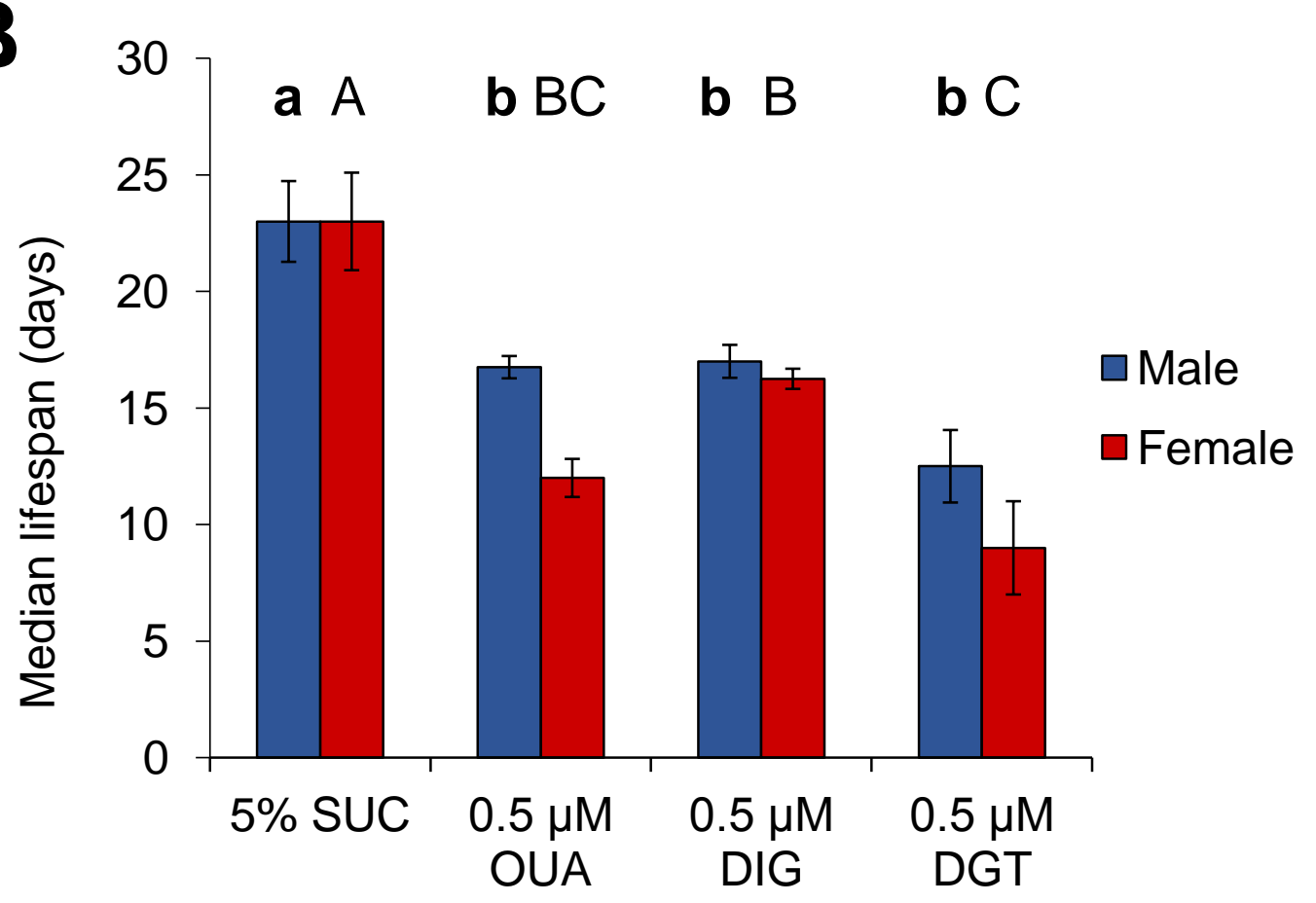

D

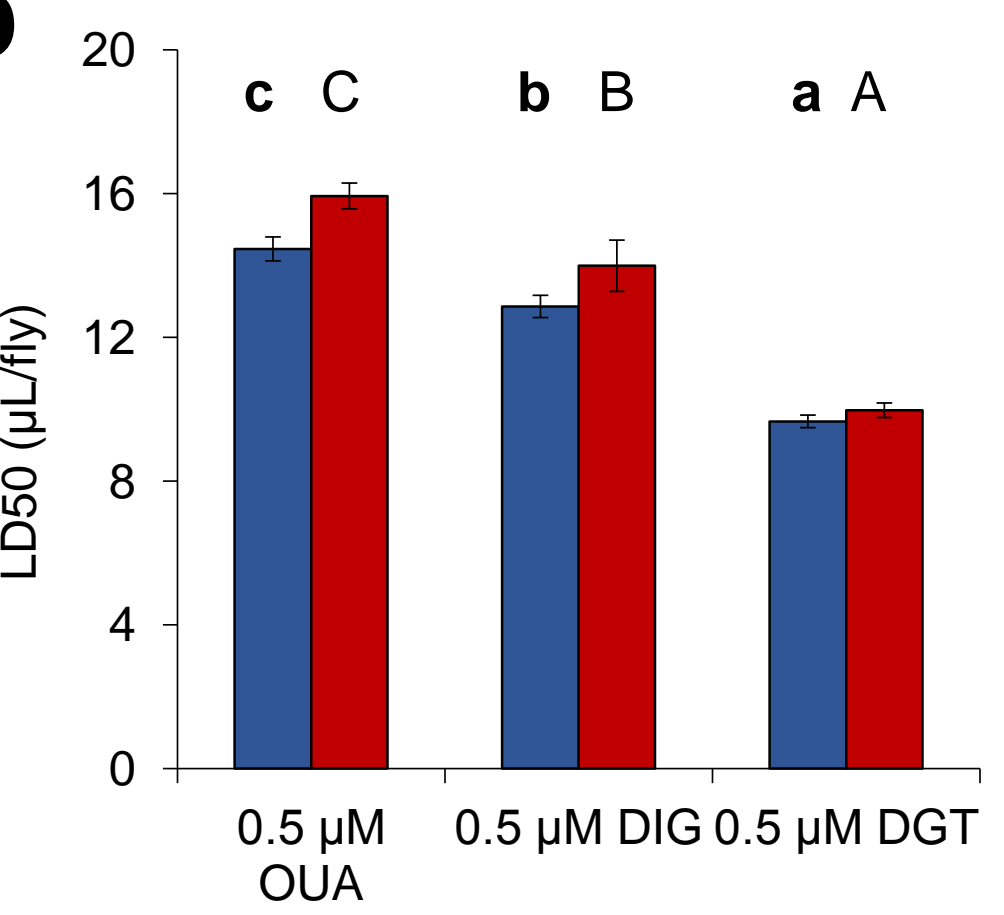



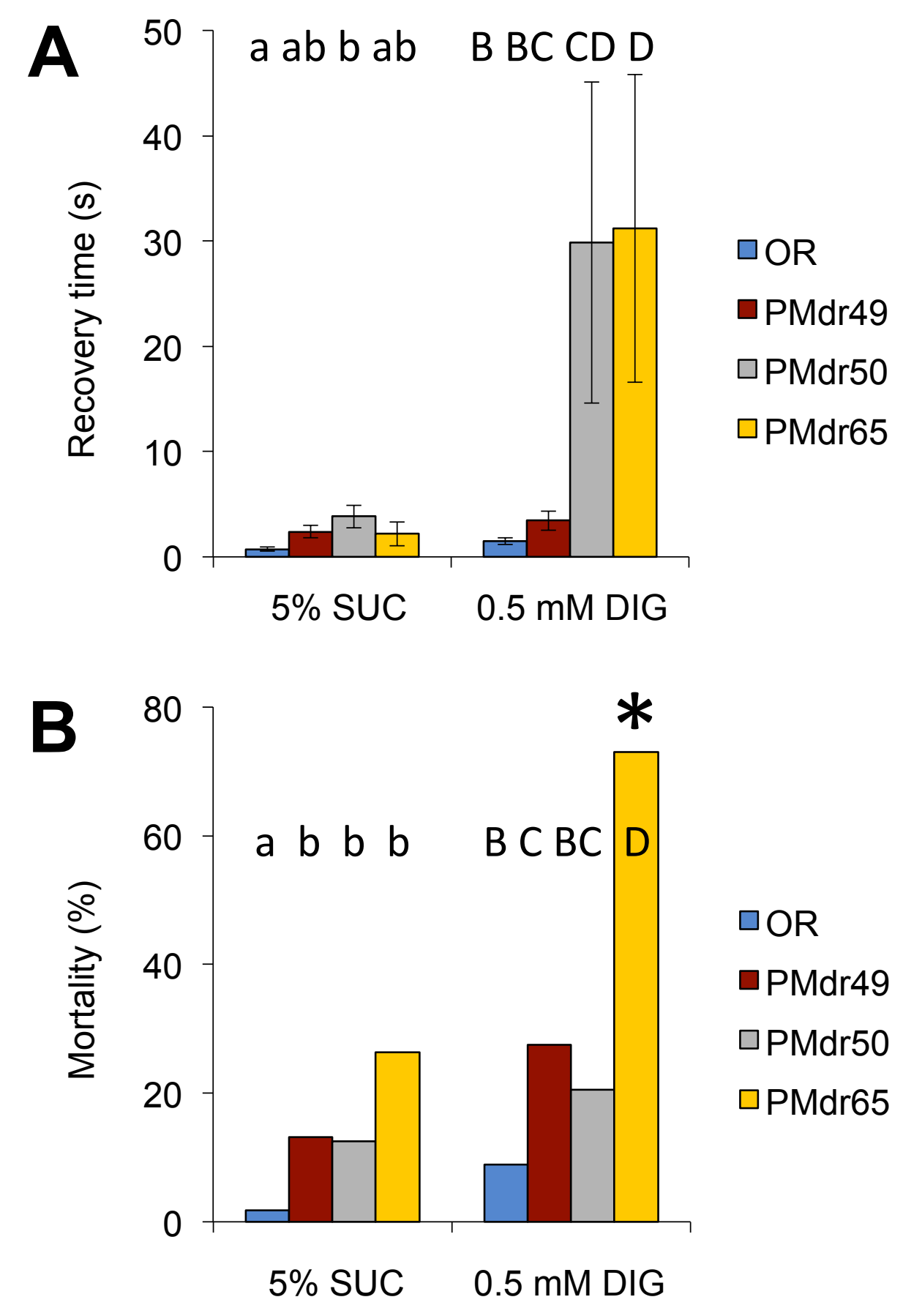


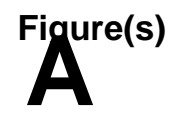

A

B

A

$A B$

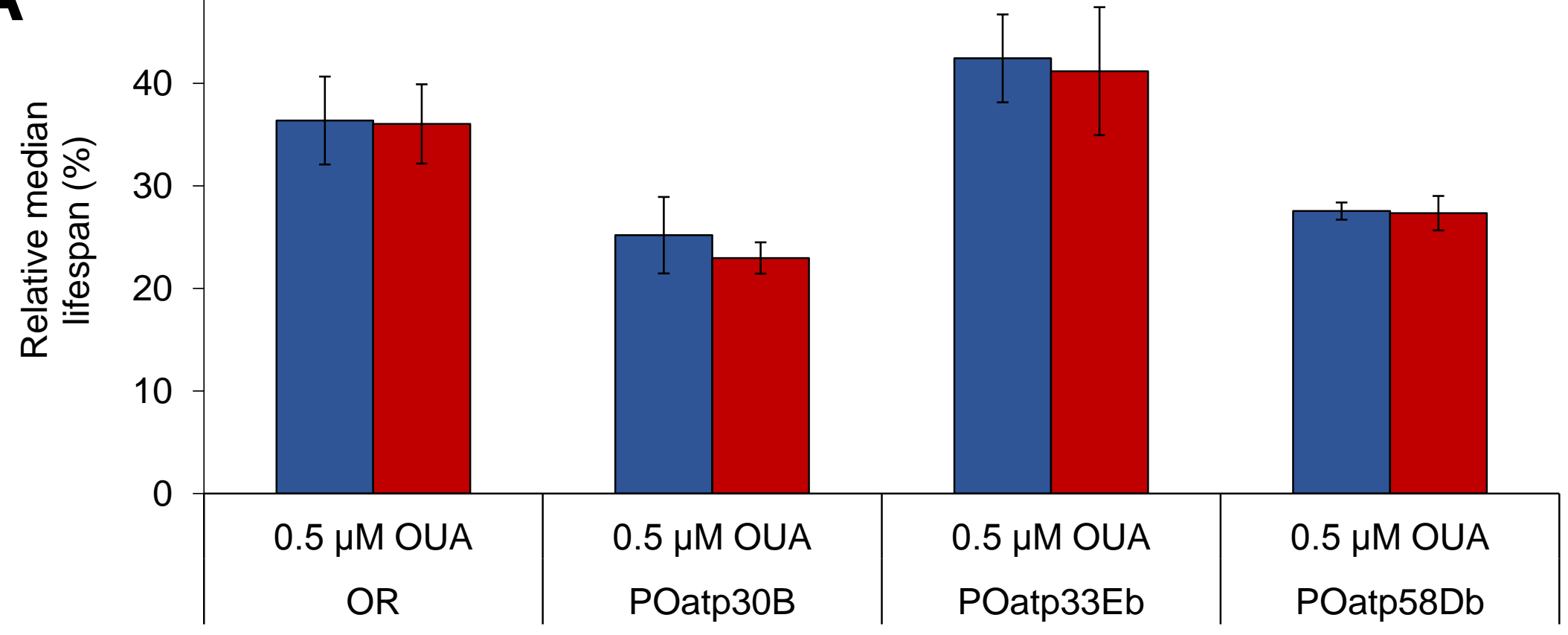

$\square$ Male

$\square$ Female

B

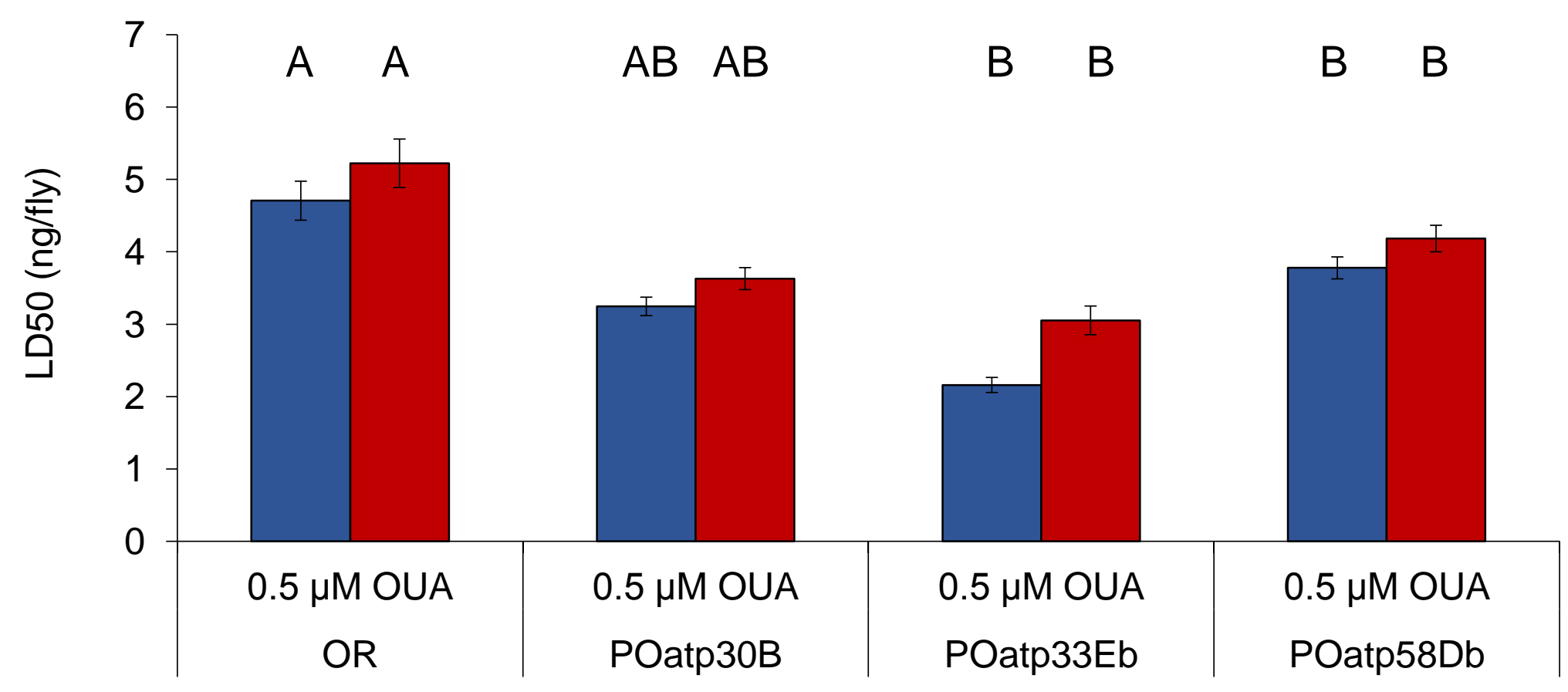

$\square$ Male

$\square$ Female 
